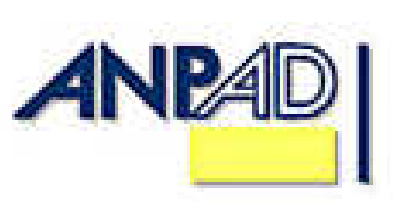

Disponível em

http://www.anpad.org.br/rac

RAC, Curitiba, v. 15, n. 5, art. 8,

pp. 931-954, Set./Out. 2011

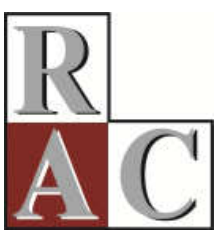

\title{
Estimando o Prêmio de Mercado Brasileiro
}

\author{
Estimating the Brazilian Market Premium
}

Walter Gonçalves Junior *

E-mail: walter.goncalves@gmail.com Fundação Getúlio Vargas - EESP/FGV São Paulo, SP, Brasil.

Ricardo Ratner Rochman

E-mail: ricardo.rochman@fgv.br Fundação Getúlio Vargas - EAESP/FGV São Paulo, SP, Brasil.

William Eid Junior E-mail: william.eid@fgv.br Fundação Getúlio Vargas - EAESP/FGV

São Paulo, SP, Brasil.

Luciana Ribeiro Chalela

E-mail: luchalela@terra.com.br Fundação Getúlio Vargas - EAESP/FGV

São Paulo, SP, Brasil

* Endereço: Walter Gonçalves Junior

Av. Nove de Julho, 2029, $2^{\circ}$ subsolo da biblioteca, São Paulo/SP, 01313-902.

Copyright (C) 2011 RAC. Todos os direitos, até mesmo de tradução, são reservados. É permitido citar parte de artigos sem autorização prévia, desde que seja identificada a fonte. 


\title{
Resumo
}

Investimentos de risco pressupõem que, em média, os ganhos sejam superiores aos obtidos por ativos livres de risco, diferencial este tradicionalmente denominado prêmio de mercado; sua importância é indiscutível para investidores nas decisões quanto à exposição aos riscos da Bolsa, para gestores de empresa, ao selecionar projetos, ou ainda para órgãos governamentais na regulação de concessionárias e na supervisão dos fundos de pensão; tal aplicabilidade demanda, entretanto, valores confiáveis para uso nos modelos. No presente trabalho são analisadas estimativas segundo três abordagens distintas, compreendendo o período de janeiro de 1996 a dezembro de 2008: na histórica, os valores encontrados variaram de 5\% a 7\% para os índices IBrX e FGV-100; na prospectiva (que reflete prêmios esperados) obteve-se 3,35\%; por fim, na indireta (ou por modelos de mercado), encontraram-se prêmios negativos, algo inesperado porém significativo.

Palavras-chave: prêmio de mercado; prêmio de risco; índices; dividendos; CAPM.

\begin{abstract}
Risky investments assume that profits are on average higher than those obtained from risk-free assets; this difference is traditionally called an equity risk premium. Its importance is unequivocal: for investors, when deciding on being exposed to the stock market's risks; for corporation managers, in project selection and even for government agencies when regulating utility company returns and supervising pension funds. However, this applicability requires trustworthy values to be used in the models. This paper analyses estimates obtained by three different approaches covering the period of January of 1996 to December of 2008. In the historical approach, the results vary from $5 \%$ to $7 \%$ for the IBrX and FGV-100 indexes; in the prospective approach (which reflects the expected premium) the result was 3.35\%; finally, in the indirect approach (by market models), negative equity premiums were found, an unexpected but significant result.
\end{abstract}

Key words: market premium; risk premium; indexes; dividends; CAPM. 


\section{Introdução}

Considerada variável de papel central para estudos e aplicações em finanças, o Prêmio de Mercado é tão intuitivo quanto sua denominação possa sugerir; de fato, costuma-se defini-lo como o diferencial requerido pelos investidores para exposição de seus recursos a ativos arriscados, em lugar dos classicamente referidos como livres de risco - estes últimos são tipicamente os títulos governamentais de curto prazo e preferencialmente indexados à inflação; portanto são os ativos com o menor risco de inadimplência a que se pode ter acesso no ambiente interno de um país, dada a reduzida exposição a fatores menos ponderáveis, tais como inflação e incertezas de longo prazo, que as demais alternativas.

Diversas são as aplicações e repercussões relativas a essa variável - do ponto de vista do investidor; a partir de suas projeções, é possível estimar retornos exigidos em aquisições de participações em empresas, ou ainda aqueles requeridos para projetos de diferentes níveis de risco; da perspectiva dos administradores de carteira e de seus cotistas, seus valores podem fornecer subsídios e referências para análises de desempenho, com consequências para a gestão dos portfólios; sob a ótica das autoridades e órgãos governamentais, pode ser útil para estabelecer retornos justos às concessionárias ofertantes de serviços com preços administrados; por fim, pode ser de interesse a todo agente ou órgão fiscalizador do sistema previdenciário complementar; haja vista os dilatados prazos de aplicação, os níveis de desempenho a serem atingidos para lograr êxito sobre as metas atuariais, bem como as profundas consequências a que estão sujeitos os beneficiários em caso de insucesso na gestão dessas carteiras.

Embora sua importância seja inquestionável, o mesmo não se pode dizer a respeito das formas pelas quais esse número vem sendo obtido e da significância dos valores encontrados - trata-se de variável não passível de observação direta, o que leva os pesquisadores e analistas de mercado a diferentes abordagens para a estimação. Como se verá no item a seguir, os resultados são muitas vezes criticados quanto ao método empregado, quanto aos valores encontrados e, a despeito até da convergência no verificado segundo diferentes abordagens e autores, quanto à consistência da fundamentação teórica a que o tema se relaciona.

Não obstante a turbulência que serve como pano de fundo para o tema, o presente trabalho tem como objetivo precípuo apresentar estimativas segundo três diferentes abordagens para o Prêmio de Mercado (ou Equity Risk Premium): em todas elas, o que se espera são respostas às seguintes perguntas de pesquisa: Há um valor significativo para o Prêmio? Qual?

\section{Revisão Bibliográfica}

À medida que modelos de apreçamento de ativos como o CAPM se disseminaram a partir da década de 80, estimativas mais apuradas para o retorno de mercado se fizeram necessárias; consequentemente, as atenções se voltaram à mensuração do Equity Risk Premium ou Prêmio de Mercado. Logo se constatou, no entanto, que a constância temporal esperada para tal variável inicialmente prevista nos primeiros modelos teóricos não se verificava. De fato, sucessivas estimações, a partir de dados históricos (estimadas a partir da diferença entre os retornos observados de uma carteira de mercado arbitrária e os de um título governamental tido como livre de risco) variavam ao longo dos anos selecionados. As contribuições mais notórias, segundo essa abordagem, são o trabalho produzido por Ibbotson Associates (1992); a sua janela histórica inicia-se em 1926 e recebeu múltiplas atualizações desde então; e o de Siegel (1992), que estendeu sobremaneira a abrangência histórica desses estudos, remonta o início das séries a 1802.

Naturalmente, outros autores ampliaram o escopo das pesquisas, ao levar as discussões ao âmbito internacional, o que permitiu a comparação entre retornos e prêmios de distintos mercados e 
realidades, sempre segundo uma perspectiva histórica; algumas interessantes contribuições, que podem ser citadas como exemplos, são Jorion e Goetzmann (1999), Canova e Nicoló (2003), Dimson, Marsh e Staunton (2003), Salomons e Grootveld (2003) e Alpalhão e Alves (2005).

Segundo essa mesma abordagem, Pastor e Stambaugh (2001) mostram também a importância de empregar séries históricas longas nessa metodologia, já que isso permite não somente redução substancial nas variâncias inerentes ao processo de estimação, mas também a possibilidade de incorporar a ocorrência de choques ou quebras estruturais presentes na amostra, levando assim a análises mais acuradas e flexíveis.

Ocorre, no entanto que, à medida que o Prêmio de Mercado passou a ser estimado e utilizado com maior frequência, começaram a surgir dúvidas quanto a sua consistência teórico-empírica e, portanto, confiabilidade. Com efeito, Mehra e Prescott (1985) pioneiramente se deram conta de que os prêmios de mercado ex post médios, estimados entre 1889 e 1978 não poderiam ser explicados senão por uma aversão relativa ao risco dos agentes incompreensivelmente alta (cerca de 40, segundo os autores, quando o esperado pelos modelos teóricos seria entre 1 e 2), resultados estes ratificados por Kocherlakota (1996) ao empregar uma base de dados ampliada em 10 anos - foi o surgimento do denominado Equity Premium Puzzle, um paradoxo entre a relativa aversão ao risco esperada para os agentes ditos racionais segundo os modelos econômicos intertemporais e os prêmios de mercado obtidos a partir de séries históricas.

Isso levou a uma fecunda (porém ainda inconclusa) linha de estudos, buscando a conciliação entre prêmios de mercado (em especial os obtidos de forma ex post) e a aversão ao risco relativo dos agentes. Uma primeira abordagem, seguida por alguns autores, foi a suposição de que, na verdade, não haveria tal paradoxo: a aversão ao risco seria, desse modo, realmente muito superior ao ditado pela teoria econômica. Ceccheti e Mark (1990) e Hansen, Sargent e Tallarini (1994) estão entre eles, embora tal visão não fosse a mais aceita, já que dela decorreria a aceitação de comportamentos (por parte dos agentes) de difícil racionalização.

Outra abordagem foi a proposição de modificações nas estruturas de preferências dos agentes. Sundersan (1989) e Constantinides (1990) sugerem o que se denominou como formação de hábito, segundo a qual a utilidade é não somente afetada por consumo presente, mas também passado. Abel (1990), Ferson e Constantinides (1991), Benartzi e Thaler (1995) e Campbell e Cochrane (1999) desenvolveram novos trabalhos segundo esses mesmos princípios, o que levou a resultados que mostram uma vinculação mais fraca entre consumo e aversão a risco. A adoção de uma função de utilidade generalizada por Epstein e Zin (1991) levou a resultados similares aos supracitados, dada a proximidade entre as ideias das duas abordagens.

Outras abordagens, visando à solução do paradoxo, se sucederam. Aiyagari e Gertler (1991), Weil (1992), Bansal e Coleman (1996), Heaton e Lucas (1996), Alvarez e Jermann (2000), Constantinides, Donaldson e Mehra (2002), McGrattan e Prescott (2005) e Storesletten, Telmer e Yaron (2007) tentaram explicá-lo a partir do relaxamento da assunção de mercados perfeitos, introduzindo imperfeições como custos de transação, restrições de crédito, fontes de risco não diversificável ou ainda impostos. Já Mankiw (1986), Constantinides e Duffie (1996), Heaton e Lucas (1997) e Storesletten et al. (2007) tentaram fazê-lo, relaxando a hipótese dos mercados completos; nenhum dos anteriores conseguiu, contudo, explicar totalmente as anomalias encontradas.

Seguindo uma linha de pensamento diversa, Rietz (1988), Danthine e Donaldson (1999) e Miller, Weller e Zhang (2002) propuseram soluções ao Puzzle, pressupondo um ambiente econômico temporariamente instável; sujeito, portanto, a severas quedas momentâneas e imprevisíveis no consumo, com desdobramentos profundos nos preços dos ativos. Essa abordagem, baseada em chances ou possibilidades de eventos adversos na economia, foi denominada Peso Problem, tendo, porém, como grande desvantagem, as dificuldades inerentes à verificação empírica.

Outra possibilidade vislumbrada para explicar a inconsistência foi o alegado viés de sobrevivência do mercado bursátil americano em relação aos demais existentes no mundo, ao longo do 
século XX, marcados por crises político-econômicas de profundos desdobramentos, assim como os flagelos de duas guerras mundiais, levando alguns deles ao fechamento temporário (o caso alemão e o japonês, por exemplo). Brown, Goetzman e Ross (1995) seguiram essa vertente. Posteriormente, a ideia foi modificada em sua essência, dando origem a uma análise baseada em ciclos econômicos e de negócios, tal como proposto por Jermann (1998), Danthine e Donaldson (1999), Boldrin, Christiano e Fisher (2001), Guvenen (2005) e também Lungu e Minford (2006).

De modo geral, Mehra (2003) argumenta que nenhuma das contribuições oriundas dessas abordagens é imune a críticas, quanto ao método ou premissas; serve também como alerta para outro problema igualmente importante: a prática de tomar como estimativa para prêmios de mercado esperados futuros a média de valores obtidos de forma retrospectiva (portanto, tomar como ex ante médias de valores ex post) - algo que por vezes se mostra traiçoeiro, como uma comparação detida entre ambos pode atestar.

Assim, é natural que, além de abordagens de cunho teórico para solução do puzzle, fossem buscadas formas para estimar o prêmio de mercado, tentando capturar seus valores esperados para o futuro, ou ex ante - teria de ser baseada, portanto, em dados de caráter prospectivo; entre outros argumentos, tem forte apelo a ideia de que prêmios negativos (tanto quanto os positivos de magnitude exageradamente elevada) constituem surpresas ao investidor, já que não são esperados retornos muito superiores ao previsto na relação tradicional entre risco e retorno esperados, bem como não haveria motivação ao investidor para se expor a ativos arriscados e registrar sistematicamente prejuízos por essa ação. Ademais, a Hipótese dos Mercados Eficientes prevê que não é possível obter vantagens ou registrar acertos sistemáticos no mercado a partir da assunção de valores passados de variáveis como estimativas justas para seus valores futuros. Freeman e Davidson (1999), entre outros, alertam para os riscos inerentes a essa prática.

Por conta disso, uma prolífica linha de trabalhos se desenvolveu tendo por base uma forma modificada do modelo de Gordon (1962) de crescimento, em que é possível estimar o retorno esperado de um ativo, a partir de variáveis presentes (preço) e projetadas (dividendos e sua taxa de crescimento). Em essência, as variáveis dividend-yield e ganho de capital são as utilizadas. Fama e French (2002) aplicaram esse modelo, tomando como ganho de capital o crescimento dos dividendos e, alternativamente, também o dos lucros, verificando que os prêmios obtidos na segunda metade do século XX superaram largamente o previsto; outro resultado relevante desse trabalho foi constatar a estacionariedade da relação dividend-yield, variável de importância central nesses estudos.

Diversos outros trabalhos se valeram também dos pressupostos do modelo de Gordon (1962), propondo nele diferentes adaptações; entre eles, citam-se Arnott e Bernstein (2002), propondo a taxa de crescimento do PNB per capita como proxy para o ganho de capital. Jagannathan, McGrattan e Scherbina (2000) documentaram uma nítida tendência de declínio do prêmio de mercado dos EUA nos últimos trinta anos, atribuível possivelmente a uma progressiva redução nas imperfeições daquele mercado.

Ainda segundo essa base conceitual, Cornell (1999) e Claus e Thomas (2001) parecem ser os pioneiros: estes últimos sugerem o uso de lucros anormais em lugar do tradicional emprego dos dividendos, usando para tanto dois estágios de crescimento no modelo de Gordon (1962); obtêm também estimativas para cinco outros países além dos EUA; abordagem similar foi adotada por Schöeder (2007), a partir de modelos de dois e três estágios para estimar os prêmios de mercado na Grã-Bretanha e Zona do Euro.

Com interesse focado no papel da inflação e em tendências assumidas pelo prêmio de mercado, Blanchard (1993) usa uma adaptação da relação dividendo-preço e constata, então, um declínio no prêmio, confirmado posteriormente por Jagannathan et al. (2000), possivelmente explicado por elevações da taxa real de juro livre de risco concomitante a quedas nos retornos reais esperados dos ativos arriscados, estas também decorrentes de inovações na inflação com efeitos de curto prazo. 
Campbell (2007) retoma o modelo de Gordon (1962) segundo uma perspectiva intemporal, abordagem esta já utilizada com sucesso, segundo o autor, por Fama e French (2002) e Pastor, Sinha e Swaminathan (2008) para estimar prêmios de mercado. Assim, a partir da relação dividendo-preço (supondo-a estacionária), o autor a manipula de modo a empregar séries de dividendo-lucro, ROE e relação book-to-market para obter estimativas do prêmio de mercado nos EUA e Canadá.

Partindo, então, desta breve revisão sobre as pesquisas internacionalmente empreendidas sobre o tema, encontram-se resumidas na Tabela 1 as estimativas de alguns autores para seis dos sete membros do G-7, o que permite observação e comparação de valores típicos em importantes países no atual cenário econômico.

Tabela 1

Prêmios de Mercado para Seis dos Países do G-7 (Vários Autores, Diferentes Períodos)

\begin{tabular}{cccccccc}
\hline Autor & EUA & Canadá & França & Alemanha & Japão & Reino Unido & Obs. \\
\hline $\begin{array}{c}\text { Dimson } \text { et al. }(2003) \\
\text { Ibbotson Associates }\end{array}$ & $4,40 \%$ & $4,00 \%$ & $3,60 \%$ & $5,70 \%$ & $5,40 \%$ & $3,80 \%$ & $\begin{array}{c}\text { Ex post, 1900-2002, em US\$, } \\
\text { real. }\end{array}$ \\
$\begin{array}{c}\text { (1992 como citado em } \\
\text { Damodaran 2001) }\end{array}$ & $6,10 \%$ & $0,68 \%$ & $2,34 \%$ & $-0,80 \%$ & $3,04 \%$ & $4,61 \%$ & $\begin{array}{c}\text { Ex post, 1970-1996, moeda } \\
\text { local, nominal }\end{array}$ \\
$\begin{array}{c}\text { Claus e Thomas } \\
(2001)\end{array}$ & $3,40 \%$ & $2,23 \%$ & $2,60 \%$ & $2,02 \%$ & $0,21 \%$ & $2,81 \%$ & $\begin{array}{c}\text { Ex ante, 1985-1998, moeda } \\
\text { local, nominal. }\end{array}$ \\
$\begin{array}{c}\text { Salomons e Grootveld } \\
(2003)\end{array}$ & $3,66 \%$ & $1,69 \%$ & $4,91 \%$ & $3,41 \%$ & $3,91 \%$ & $4,41 \%$ & $\begin{array}{c}\text { Ex post, 1976-2001 (ao ano, em } \\
\text { US\$, nominal) }\end{array}$ \\
$\begin{array}{c}\text { Canova e Nicoló } \\
(2003)\end{array}$ & $5,68 \%$ & $2,26 \%$ & $4,12 \%$ & $3,19 \%$ & $3,63 \%$ & $5,15 \%$ & $\begin{array}{c}\text { Ex post, 1971-1999, moeda } \\
\text { local, real } \\
\text { Pastor } \text { et al. (2008) }\end{array}$ \\
$2,60 \%$ & $4,70 \%$ & $2,90 \%$ & $1,60 \%$ & $3,10 \%$ & $2,90 \%$ & $\begin{array}{c}\text { Ex ante, 1990-2002, moeda } \\
\text { local, nominal. }\end{array}$ \\
\hline
\end{tabular}

Nota. Fonte: Elaboração própria.

No Brasil, diversos estudos, como Bonomo e Domingues (2002), Alencar (2002), Soriano (2002), Sampaio (2002), E. A. Araújo (2005), Catalão e Yoshino (2006) e Santos (2006) apontaram uma possível inexistência do chamado Equity Premium Puzzle, divergindo, portanto, das conclusões de Cysne (2005); em parte, como alertado por Goulart e Paiva (2005) e A. M. P Araújo, Assaf e Lima (2006), os autores podem ter esbarrado nas conhecidas dificuldades em estimar um prêmio de risco com confiabilidade em vista da qualidade das informações disponíveis e da forte volatilidade presente no mercado brasileiro de capitais, razão pela qual Minardi e Sanvicente (2006) sugerem tal estimação pela abordagem prospectiva ou implícita, utilizando para tanto o modelo de dividendos descontados. Eles observam, também, menor volatilidade da série de prêmios esperados, quando comparada à dos realizados, algo provavelmente explicável pela oscilação da relação dividendo-preço ser negativamente relacionada à variação do PIB.

Não obstante tais dificuldades, há contribuições que apontam valores objetivos para o prêmio de mercado. Corrêa e Samanez (2002) encontraram um prêmio pelo risco brasileiro ex post de 9\%; Leal (2002) estimou o prêmio de mercado brasileiro (em dólares) em 8,2\% no período de 1974 a 2001, ressalvando-se porém que em 168 meses dessa amostra (cerca de 50\%, portanto) o prêmio de risco foi negativo. Já Bonomo e Garcia (1994) encontraram um prêmio de risco de 5,3\%, enquanto seus modelos com variável aleatória de consumo e taxas de crescimento de dividendo apresentaram um valor próximo a $2,5 \%$. 


\section{Metodologia}

No presente trabalho, iremos estimar o prêmio de mercado segundo três abordagens distintas: a histórica ou retrospectiva, através de dados históricos dos índices de mercado e de ativos considerados livres de risco; a implícita ou prospectiva, através de dados históricos de dividendos de várias ações negociadas na Bovespa; e a indireta, através do previsto no modelo CAPM. Elas são descritas separadamente, conforme segue.

\section{Abordagem Retrospectiva ou Histórica}

Para esta abordagem foram selecionadas as cotações históricas diárias de fechamento nominais e reais (corrigidas por dividendos e proventos) de três dos índices de mercado mais usados: o Ibovespa (entre janeiro de 1996 e dezembro de 2008, o que totaliza 13 anos de dados); o IBrX (remontando de janeiro de 1996 a dezembro de 2008, o que também totaliza 13 anos de dados); e o FGV-100, referenciado à mesma janela (de janeiro de 1996 a dezembro de 2008). Tal princípio se justifica, especialmente em função de os primeiros registros de cotações do $\mathrm{IBrX}$ ocorrerem naquele mês, tornando assim as estimativas dos três índices comparáveis; no entanto esse corte é igualmente conveniente, se lembrarmos que ele toma somente valores posteriores ao lançamento do Plano Real (em julho de 1994), evento este que encerrou um período de realidade hiperinflacionária no Brasil, o que descarta, portanto, observações contaminadas por tal ambiente desestruturado.

Assim, a estimativa do prêmio de mercado neste caso passa a ser direta e intuitiva: consiste em obter médias para o retorno de mercado e para o ativo livre de risco para, na sequência, efetuar-se a diferença entre ambas.

O tradicional problema enfrentado nesse tipo de abordagem é apropriar um banco de dados com frequência e abrangência adequados, limitando assim os erros inerentes às estimativas a patamares aceitáveis - normalmente, o recomendável é o emprego de séries anuais (compreendendo o maior período possível), por serem mais estáveis e menos sujeitas à volatilidade e ao nervosismo de curtíssimo prazo dos mercados; para esses casos, conforme alertado por Jorion e Goetzmann (1999), Pastor e Stambaugh (2001) e Damodaran (2001), o recomendável é que as séries possuam uma quantidade de observações tal que seu erro-padrão seja pouco representativo perante a média a ser estimada.

Nos EUA, autores como Siegel (1992), Arnott e Bernstein (2002), entre outros, seguiram esse princípio, recorrendo a séries bastante longas que, por vezes, remontaram até dois séculos anteriores à data de estudo; no Brasil, isso é claramente inviável não somente pela relativa juventude de nossos mercados, em face do seu par americano, entre outros, como também pela baixa representatividade de informações perturbadas por cenários hiperinflacionários e de crises internas.

Isso nos leva, invariavelmente, ao uso de séries mensais de retornos que, embora de sobejo mais voláteis que as anuais, propiciam a construção de uma amostra com numerosidade minimamente compatível com as estimativas a serem feitas (em função dos desvios inerentes). Vale mencionar, não obstante, o fato de que Jorion e Goetzmann (1999) mencionam que séries desse tipo levam a melhores e mais precisas estimativas, além de serem versáteis para a condução de outros testes adicionais (para estudos de evento, ou a inserção de dummies para a captação de efeitos devidos a quebras estruturais).

Assim, a partir das cotações diárias dos três índices de mercado supra e do CDI, é possível obter os respectivos retornos médios mensais de mercado e livres de risco das diversas observações, tendo como referência sempre o primeiro dia útil da cada mês da amostra. Na sequência, para obter a estimativa desejada do prêmio de mercado há duas possibilidades. 
Cálculo aritmético: é possível mensalmente calcular o retorno da carteira de mercado e do ativo livre de risco (ambos em taxa equivalente ao dia útil, a partir dos intervalos definidos entre cada primeiro dia útil de cada mês da amostra); em seguida, basta estimar a média aritmética desses retornos, resultado que é finalmente anualizado, com base de 252 dias úteis, para facilidade de apreciação e comparação. Cabe alertar que convém realizar a anualização após o cálculo da média, o que evita maior propagação de erros; por fim, o prêmio pelo risco é representado pela diferença entre ambos.

- Cálculo geométrico: diversamente, é possível também verificar o retorno acumulado em todo o período, tanto da carteira de mercado quanto do ativo livre de risco, estimando-se que o prêmio poderia ser representado pela diferença entre essas duas médias, devidamente anualizadas pelo total de dias úteis do intervalo.

\section{Abordagem Prospectiva ou Implícita}

Outra forma de estimar o prêmio pelo risco seria observar as expectativas do mercado a respeito dos retornos esperados para os ativos no futuro. Vários autores veem essa abordagem como mais promissora não somente no que se refere aos problemas relativos ao Equity Premium Puzzle como também uma solução da controvérsia (no caso dos EUA) de que não se pode esperar que os prêmios passados venham a se repetir consistentemente no futuro.

Assim, a exemplo de Fama e French (2002) e Campbell (2007), será utilizada uma forma intemporal do Modelo de Gordon (1962) modificado, conforme segue.

$$
\mathrm{P}_{0, \mathrm{i}}=\frac{\mathrm{D}_{1, \mathrm{i}}}{\mathrm{r}_{\mathrm{i}}-\mathrm{g}_{\mathrm{i}}} \Leftrightarrow \mathrm{r}_{\mathrm{i}}=\frac{\mathrm{D}_{1, \mathrm{i}}}{\mathrm{P}_{0, \mathrm{i}}}+\mathrm{g}_{\mathrm{i}}
$$

Onde:

$\mathrm{P}_{0, \mathrm{i}} \quad=$ Valor do ativo i na data zero;

$\mathrm{D}_{1, \mathrm{i}} \quad=$ Dividendo a ser distribuído pelo ativo i no próximo período (data um);

$\mathrm{r}_{\mathrm{i}} \quad=$ Retorno esperado para o ativo $\mathrm{i}$;

$\mathrm{g}_{\mathrm{i}} \quad$ = Ganho de capital esperado para o ativo i;

Note-se que, a exemplo do que os autores supra fizeram em seus trabalhos, também será considerada a relação $\mathbf{D}_{\mathbf{1}, \mathbf{i}} / \mathbf{P}_{\mathbf{0}, \mathbf{i}}$ (normalmente referida na literatura como dividend yield) como estacionária, portanto a ser obtida a partir da média dos valores disponíveis para cada ativo selecionado.

Quanto ao ganho de capital ou taxa de crescimento $\left(\mathbf{g}_{\mathbf{i}}\right)$, há algumas sugestões na literatura acadêmica sobre a melhor proxy: podem-se estimar diretamente as taxas médias de crescimento dos dividendos ou ainda dos lucros de onde eles se originam. Autores como Campbell (2007) sugerem métricas menos sujeitas à discricionariedade dos administradores (dividendos são subordinados às políticas de distribuição, com não raros conflitos de agência inerentes), tais como tomar pelo crescimento a taxa de reinvestimento (o produto da taxa de retenção de lucros pelo ROE da empresa). Arnott e Bernstein (2002) defendem o emprego da taxa de crescimento do PNB per capita descontada de diluições ou perdas da série de dividendos em relação ao restante da economia do país. No presente trabalho, para evitar os problemas citados com as políticas de distribuição (e sua erraticidade no Brasil quanto a valores aprovados e periodicidade), adotaremos as taxas de reinvestimento como 
representativas dos diversos $\mathbf{g}_{\mathbf{i}}$, supondo-os ainda estáveis (portanto superadas eventuais fases de rápido crescimento nas empresas).

Para as estimativas a serem realizadas segundo (1) foi inicialmente selecionado um banco de dados disponível no site da Bovespa, no qual constam mais de 10.000 registros de dividendos e proventos diversos de 318 empresas, desde janeiro de 1996 a dezembro de 2008. Após descartadas 160 empresas por insuficiência de dados ou informações para os cálculos, além de outras 27 por serem instituições financeiras, as 131 remanescentes (conforme relacionadas na Tabela 2) tiveram seus proventos em datas coincidentes consolidados, permitindo assim a ocorrência de somente uma única distribuição por data.

Tabela 2

\section{Ativos Selecionados para a Estimação do Retorno de Mercado Brasileiro Segundo a Abordagem ex ante}

\begin{tabular}{cccccccccccc}
\hline ABNB3 & ALLL4 & ALPA4 & AMBV4 & ARCZ6 & AVIL3 & BDLL4 & BMTO4 & BPIA3 & BRAP4 & BRKM5 & BRTO4 \\
BRTP4 & CCRO3 & CEEB3 & CEPE5 & CGAS5 & CGRA4 & CLSC3 & CMIG4 & CNFB4 & COCE5 & CPFE3 & CPFP4 \\
CPLE6 & CRUZ3 & CSAB4 & CSMG3 & CSNA3 & CSRN3 & CTKA4 & CTNM4 & CYRE3 & DOHL4 & DROG3 & DUQE4 \\
DURA4 & DXTG4 & EBTP4 & ELET3 & EMBR3 & ENER6 & ESCE3 & ETER3 & FBMC4 & FESA4 & FFTL4 & FJTA4 \\
FRAS4 & GEPA3 & GETI4 & GGBR4 & GLOB3 & GOAU4 & GOLL4 & GUAR3 & IGUA6 & ITEC3 & ITSA4 & JFEN3 \\
KEPL3 & KLBN4 & LAME4 & LECO4 & LEVE4 & LREN3 & LUXM4 & MFLT4 & MGEL4 & MOAR3 & MRSL4 & MTSA4 \\
NAFG4 & NATU3 & NEMO4 & PALF3 & PATI4 & PCAR4 & PEAB4 & PETR4 & PNVL3 & POMO4 & PQUN3 & PRGA3 \\
PSSA3 & PTNT4 & PTPA4 & RAPT4 & RCSL4 & RENT3 & RGEG3 & RNPT4 & ROMI3 & RPAD3 & RPMG4 & RSID3 \\
RSUL4 & SAPR4 & SBSP3 & SCAR3 & SDIA4 & SGEN4 & SHUL4 & SJOS4 & SLED4 & SOND6 & SPRI3 & SULT4 \\
SUZB5 & TBLE3 & TCNO4 & TCSL4 & TIBR5 & TKNO4 & TLPP4 & TMAR5 & TMCP4 & TMGC3 & TNCP4 & TNLP4 \\
TRPL4 & TXRX4 & UCOP4 & UGP44 & UNIP6 & USIM5 & VALE5 & VCPA4 & VGOR4 & WEGE3 & WHRL4 & \\
\hline
\end{tabular}

Nota. Fonte: Elaboração própria.

As cotações dos ativos $\left(\mathbf{P}_{\mathbf{0}, \mathbf{i}}\right)$ empregadas em (1) foram sempre nominais, selecionadas para a data anterior mais próxima o possível da assembléia onde ocorreu a divulgação do dividendo/ rendimento/ JCP considerado, lembrando-se que para a coerência das estimativas as cotações precisaram ser corrigidas (somente) para os casos de agrupamentos e desdobros. Uma vez obtidos os retornos esperados para vários ativos selecionados segundo (1), o retorno da carteira de mercado poderia ser estimado a partir da sua média simples ou, mais apropriadamente, a partir da média ponderada pelos patrimônios líquidos de cada empresa, conforme expresso a seguir:

$$
\mathrm{R}_{\mathrm{m}}=\frac{\sum \mathrm{PL}_{\mathrm{i}} \mathrm{r}_{\mathrm{i}}}{\sum \mathrm{PL}_{\mathrm{i}}}
$$

Onde:

$\mathrm{R}_{\mathrm{m}} \quad=$ Retorno esperado para a carteira de mercado;

$\mathrm{PL}_{\mathrm{i}} \quad=$ Patrimônio líquido mais atual da empresa $\mathrm{i}$;

$\mathrm{r}_{\mathrm{i}} \quad=$ Retorno esperado para a empresa $\mathrm{i}$;

Vale a ressalva de que nos casos isolados, em que o patrimônio líquido se apresentou negativo, a observação foi desconsiderada. Note-se ainda que aparecem em destaque na Tabela 2 os ativos cujos retornos apresentaram valores atípicos em relação à média da amostra, ou seja, cujo afastamento foi 
superior a dois desvios-padrão, o que motivou o cálculo em (2) com e sem esses ativos, visando conferir robustez à estimação.

Vencido esse passo, o prêmio de mercado pode ser estimado como a diferença entre esse retorno médio de mercado e o retorno esperado futuro (médio) para o ativo livre de risco. Para tanto, é possível usar uma média das taxas implícitas dos contratos futuros DI 1 dia com maturidades as mais longas que for possível, abertos em dezembro de 2008 (limite da amostra), compatibilizando-se assim com o disposto em (1), para extrair tal estimativa.

\section{Abordagem por Modelos de Mercado ou Indireta}

Uma terceira via para estimar o prêmio de mercado é usar a SML (Security Market Line), que deriva do previsto no CAPM a partir de uma perspectiva temporal:

$$
\mathrm{R}_{\mathrm{t}}-\mathrm{Rf}_{\mathrm{t}}=\alpha \mathrm{i}+\left(\mathrm{Rm}_{\mathrm{t}}-\mathrm{Rf}_{\mathrm{t}}\right) \cdot \beta \mathrm{i}+\varepsilon^{\mathrm{i}_{\mathrm{t}}}
$$

Diferentemente do convencional, se considerarmos como variável explicativa da especificação o termo $\beta^{\mathrm{i}}$, então a diferença $\mathrm{Rm}_{\mathrm{t}}-\mathrm{Rf}_{\mathrm{t}}$ (nitidamente o prêmio de mercado que buscamos) seria a inclinação da reta ajustada; um eventual intercepto dessa mesma regressão, se significativo, seria um retorno anormal produzido pelo ativo em análise.

Tal estimativa seria possível mediante uma seleção de ativos com liquidez suficiente para formar uma amostra numerosa o suficiente para sustentar tais testes; tomaram-se então as cotações diárias nominais de fechamento (corrigidas por dividendos e proventos diversos) compreendidas entre janeiro de 1996 e dezembro de 2008 dos ativos relacionados na Tabela 3.

Tabela 3

Ativos Selecionados para a Estimação do Prêmio de Risco Brasileiro

\begin{tabular}{ccccccccccccc}
\hline ACES4 & ALPA4 & AMBV4 & ARCE3 & ARCZ6 & BBAS3 & BBDC4 & BOBR4 & BRAP4 & BRKM5 & BRTO4 & BRTP4 & CCRO3 \\
CESP5 & CGAS5 & CLSC6 & CMET4 & CMIG4 & CNFB4 & CPLE6 & CRTP5 & CRUZ3 & CSNA3 & CTNM4 & DURA4 & EBTP4 \\
ELET6 & ELPL5 & EMBR3 & ETER3 & FFTL4 & FJTA4 & GETI4 & GGBR4 & GOAU4 & GUAR3 & ITUB4 & ITSA4 & KLBN4 \\
LAME4 & LEVE4 & LIGT3 & MAGS5 & MYPK4 & NETC4 & PALF3 & PCAR5 & PETR4 & PMAM4 & POMO4 & PRGA4 & PTIP4 \\
RAPT4 & RHDS3 & RPSA4 & RSID3 & SBSP3 & SDIA4 & SLED4 & SUZB5 & SZPQ4 & TBLE3 & TCOC4 & TCSL4 & TLCP4 \\
TLPP4 & TMAR5 & TMCP4 & TNLP4 & TRPL4 & WEGE4 & UGPA4 & UNIP6 & USIM5 & VALE5 & VCPA4 & VIVO4 & VAGV4 \\
\hline
\end{tabular}

Nota. Fonte: Elaboração própria.

Neste trabalho, para a condução da regressão especificada em (3) diversos cuidados metodológicos visando consistência (e evitando tendenciosidades) se fizeram necessários.

- Subdivisão da amostra das cotações: para evitar que as cotações de fechamento diárias dos ativos selecionados (das quais são obtidos os retornos) fossem duplamente utilizadas em regressões (ou inferências) sequenciais ou dependentes, a base que contém as cotações de todos os ativos foi dividida em duas subamostras: a das linhas de cotações de ordem par e as de ordem ímpar. Os vetores de observações de ordem impar foram destinados somente à estimação das variáveis explicativas a serem empregadas nos testes (os betas do CAPM), enquanto as de ordem par foram usadas somente na obtenção das variáveis dependentes (retornos excedentes) nos testes inerentes à regressão especificada em (3). 
A estimação prévia dos $\beta^{\mathbf{i}}$ : conforme descrito no passo anterior, das observações de ordem impar são extraídas as estimativas dos $\beta^{i}$ (beta segundo o CAPM) de cada ativo selecionado. É possível realizar semelhante estimação, a partir da especificação (3), em que a variável dependente é o retorno excedente de cada ativo, e a explicativa é o retorno excedente de mercado, ambos expressos em taxa efetiva ao dia útil. Das séries históricas assim formadas foram expurgadas as observações consideradas atípicas: as de afastamento superior a três desvios-padrão da média anual imediatamente anterior. Por fim, como proxies da carteira de mercado e do ativo livre de risco foram adotados, respectivamente, o Ibovespa e o CDI; a janela móvel mensal de estimação, adotada para cada $\beta$, foi de três anos, visando assim ao melhor aproveitamento da amostra (reduzir a perda de observações no próximo passo).

Retornos excedentes: para representar o retorno passado médio de um dado ativo a cada mês, definiu-se como tal o respectivo retorno acumulado dos últimos 12 meses, expresso ao dia útil (compatibilizado assim à unidade adotada para a estimação dos betas), empregando para tanto, como já mencionado, subamostra com as cotações de ordem par.

- Modelo teórico base: a variável explicativa (betas), por construção, pode levar a séries temporais altamente persistentes (especialmente se seguida a recomendação tradicional de estimação a partir de cinco anos de cotações); além do fato de que, caso ela não seja suficientemente longa pode resultar não estacionária. Para lidar com o potencial problema gerado pelo segundo desvio das premissas tradicionais para regressões lineares, haveria duas possibilidades: selecionar ativos cujas séries de $\beta^{i}$ e retornos excedentes sejam estacionários, ou tentar trabalhar com as primeiras diferenças dessas séries.

Nesse último caso, postulando-se que a variável de interesse $r_{m}$ (o prêmio médio esperado de mercado) seja invariável nas dimensões tempo e cross section, então $\mathrm{Rm}-\mathrm{Rf}=\mathrm{r}_{\mathrm{m}}$; denominando-se ainda o retorno excedente de cada ativo como $\mathrm{r}_{\mathrm{t}} \mathrm{H}=\mathrm{R}_{\mathrm{t}}-\mathrm{Rf}_{\mathrm{t}}$ e diferenciando-se (3) teríamos:

$$
\Delta \mathrm{r}_{\mathrm{t}}=\gamma^{\mathrm{i}}+\mathrm{r}_{\mathrm{m}} \cdot \Delta \beta \mathrm{i}_{\mathrm{t}}+\eta \mathrm{i}_{\mathrm{t}}
$$

Método de regressão: independentemente do discutido acima como modelo teórico base, ao utilizarmos retornos na obtenção dos $\beta^{i}$ e deles nos valermos novamente como variável dependente leva a uma especificação em que a variável explicativa e dependente são geradas por um mesmo processo estatístico, o que as tornaria endogenamente relacionadas, afrontando portanto algumas das premissas básicas do modelo de MQO; a despeito das providências adotadas com relação às cotações que geram os retornos, ignorar-se o fato e empregar regressões tradicionais por MQO levariam a estimativas potencialmente enviesadas, tendenciosas e inconsistentes. Nesses casos, métodos mais genéricos e abrangentes, como GMM (Método dos Momentos Generalizados) se fazem necessários, embora um natural obstáculo ao seu emprego seja a definição de instrumentos adequados, ou seja, variáveis instrumentais suficientemente correlacionadas com a variável explicativa $(\beta \mathrm{i})$, mas mantendo baixa correlação com os resíduos decorrentes da regressão principal ( 3 ou 4, conforme o caso); no presente trabalho, foram selecionadas como tal as séries dos próprios betas (ou de suas diferenças); porém seriam defasadas em um mês das datas-referência. Por fim, vale ressaltar que se trata de uma regressão em painel: séries temporais mensais dos retornos excedentes, ou de suas diferenças, de 78 ativos da Bovespa como variável dependente e as respectivas séries temporais mensais dos seus betas ou diferenças; aí seriam testados efeitos fixos e aleatórios temporais para o intercepto, já que não se esperam efeitos fixos ou aleatórios cross section no presente caso, isto é, variações da taxa livre de risco por ativo não são esperadas, embora se admita o caso na dimensão tempo.

Explicada assim a preparação dos dados e seleção dos métodos empregados, resta declarar o foco desta sessão, que é em essência o mesmo objetivo das outras duas sessões anteriores: a estimativa do prêmio de mercado, que de forma mais objetiva consistiria na rejeição (ou não) da hipótese nula $\mathbf{H}_{\mathbf{0}}: \mathbf{r}_{\mathbf{m}}=\mathbf{0}$, ou seja, se é possível mensurar de forma significativa um valor para tanto. 
Uma vez explicitadas as três abordagens aqui empregadas na estimação do prêmio de mercado (métodos e dados), passemos aos resultados encontrados.

\section{Resultados}

Preliminarmente aos resultados para o prêmio propriamente dito, é ilustrativo apresentar o comportamento do mercado (representado por seus índices mais comuns) e do ativo livre de risco durante o período definido para análise; é o que segue expresso na Figura 1, onde se observam retornos segundo médias móveis anuais estimadas mês a mês (diferentemente dos retornos usados nas estimativas do prêmio, portanto). Suavizam-se assim as variações expressivas que ocorreram, facilitando a visualização e identificação dos comportamentos.

Nele são notáveis as fortes altas no final da década de 90 e em 2004, bem como o período de estagnação entre 2000 e 2002 e o de crise a partir de maio de 2008. Qualitativamente também é evidente a coerência entre os três índices selecionados; os resultados obtidos em termos reais (descontados portanto os efeitos inflacionários), embora não expressos aqui em função da exiguidade do espaço disponível, são bastante similares.

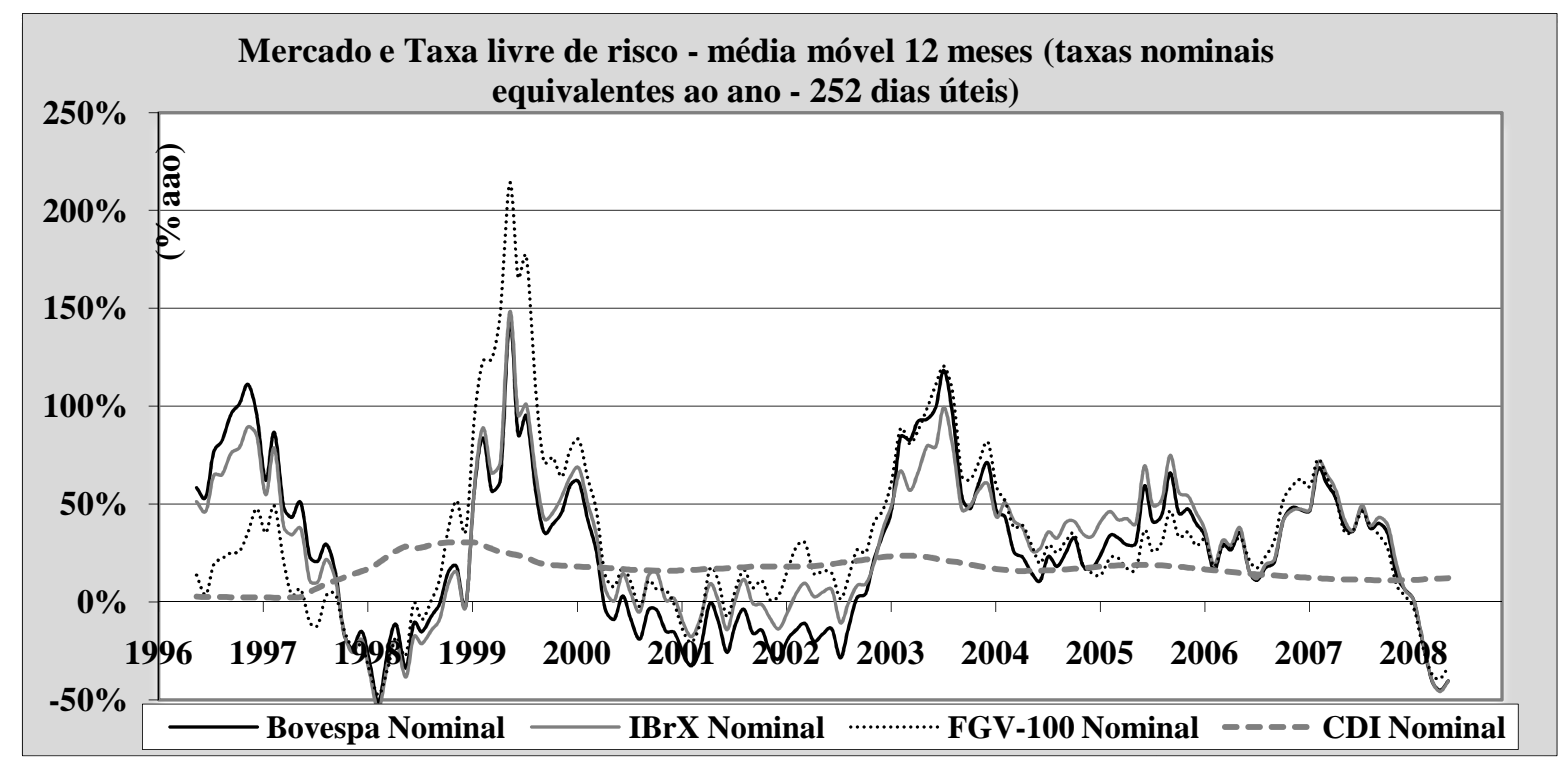

Figura 1. Retornos Médios Anuais dos Índices de Mercado Selecionados e do Ativo Livre de Risco (CDI).

Fonte: Elaboração própria.

Assim, o passo seguinte é apresentar as estimativas históricas obtidas para os índices em questão; a Tabela 4 mostra as estatísticas descritivas e testes de estacionariedade decorrentes do cálculo aritmético, onde se vê exceto no Ibovespa real que todas as médias são significativas a probabilidades inferiores a $15 \%$ para um total de 156 observações, decorrentes dos 13 anos do intervalo usado, com meses de normalmente 21 dias úteis. Como esperado, apenas o CDI nominal apresenta possibilidade de tendência, o que indicaria declínio da taxa no período. 
Tabela 4

Resumo das Estatísticas Descritivas e Testes de Estacionariedade, Dados Ex Post dos Retornos da Carteira de Mercado e da Taxa Livre de Risco (CDI) para Diferentes Índices, Cálculo Aritmético

\begin{tabular}{|c|c|c|c|c|c|c|c|c|c|}
\hline \multirow[b]{2}{*}{ tins } & \multirow{2}{*}{$\begin{array}{l}\text { Dias } \\
\text { úteis }\end{array}$} & \multicolumn{2}{|c|}{ Bovespa } & \multicolumn{2}{|c|}{ IBrX } & \multicolumn{2}{|c|}{ FGV-100 } & \multicolumn{2}{|c|}{$\overline{\mathrm{CDI}}$} \\
\hline & & Nominal & Real & Nominal & Real & Nominal & Real & Nominal & Real \\
\hline (ód & 20,63 & $72 \%$ & $0,045 \%$ & $083 \%$ & $0,056 \%$ & $0,085 \%$ & $0,059 \%$ & $0,059 \%$ & $0,033 \%$ \\
\hline Mediana & 21,00 & 0,09070 & $0,071 \%$ & $0,129 \%$ & $0,096 \%$ & $0,104 \%$ & $0,082 \%$ & $0,061 \%$ & $0,034 \%$ \\
\hline Máximo & 2300 & $1,312 \%$ & $1,274 \%$ & $1,138 \%$ & $1,100 \%$ & $1,172 \%$ & $1,124 \%$ & $0,141 \%$ & $0,137 \%$ \\
\hline Mír & 18,00 & -1 & $1, \overline{-}$ & -1 & $\stackrel{-}{1,952 \%}$ & $-1,3$ & $1,287 \%$ & $0,008 \%$ & $0,072 \%$ \\
\hline Desvi & 35 & $0,479 \%$ & $0,475 \%$ & $0,442 \%$ & $0,439 \%$ & $0,392 \%$ & $0,388 \%$ & $0,028 \%$ & $0,035 \%$ \\
\hline Prob: & $0,00 \%$ & $6,40 \%$ & $23,80 \%$ & $2,10 \%$ & $11,24 \%$ & $0,75 \%$ & $6,15 \%$ & $0,00 \%$ & $0,00 \%$ \\
\hline Assime & $-0,21$ & $-0,0 \mathrm{~J}$ & $-0,00$ & $-0,0$ & $-0,85$ & $-0,29$ & $-0,28$ & 0,15 & $-0,12$ \\
\hline Curtose & 2,45 & 4,39 & 4,26 & 5,46 & 5,25 & 3,90 & 3,77 & 3,67 & 4,09 \\
\hline Jarque-Bera & 3,128 & 22,775 & 19,684 & 59,049 & 50,911 & 7,559 & 5,901 & 3,387 & 8,141 \\
\hline Probabilidade & $20,93 \%$ & $0,00 \%$ & $0,01 \%$ & $0,00 \%$ & $0,00 \%$ & $2,28 \%$ & $5,23 \%$ & $18,39 \%$ & $1,71 \%$ \\
\hline & - & $-6,43$ & $-6,43$ & $-6,26$ & $-6,282$ & $-5,983$ & $-5,985$ & $-2,695$ & $-3,169$ \\
\hline & - & $0,00 \%$ & $0,00 \%$ & $0,00 \%$ & $0,00 \%$ & $0,00 \%$ & $0,00 \%$ & $24,04 \%$ & $9,46 \%$ \\
\hline & - & $-12,094$ & $-12,247$ & $-12,087$ & $-12,251$ & $-10,768$ & $-10,926$ & $-2,533$ & $-3,942$ \\
\hline & - & $0,00 \%$ & $0,00 \%$ & $0,00 \%$ & $0,00 \%$ & $0,00 \%$ & $0,00 \%$ & $31,19 \%$ & $1,26 \%$ \\
\hline Observações & 156 & 156 & 156 & 156 & 156 & 156 & 156 & 156 & 156 \\
\hline
\end{tabular}

Conforme descrito em Abordagem Retrospectiva ou Histórica, essas médias, após anualizadas, permitem a estimativa do prêmio de mercado por índice (segundo o cálculo aritmético), algo que pode ser confrontado com o respectivo cálculo geométrico, conforme demonstrado na Tabela 5, na sequência.

Tabela 5

Prêmios de Risco Ex Post para Diferentes Índices e Abordagens Selecionadas.

\begin{tabular}{lcccccc}
\hline & \multicolumn{2}{c}{ Bovespa } & \multicolumn{2}{c}{ IBrX } & \multicolumn{2}{c}{ FGV-100 } \\
& Nominal & Real & Nominal & Real & Nominal & Real \\
\hline Aritmética & $3,66 \%$ & $3,42 \%$ & $7,05 \%$ & $6,59 \%$ & $7,80 \%$ & $7,30 \%$ \\
Geométrica & $2,25 \%$ & $2,10 \%$ & $5,75 \%$ & $5,38 \%$ & $6,76 \%$ & $6,33 \%$ \\
\hline
\end{tabular}

É interessante registrar certa tendência de os valores aritméticos serem superiores aos geométricos, bem como a maior proximidade entre as estimativas oriundas dos índices mais amplos e estáveis (em termos de constituição e revisão), a despeito da abordagem de estimação selecionada. É natural que tais resultados nos induzam a apontar os valores destes dois índices (IBrX e FGV-100), em termos reais, como os mais prováveis em representar o prêmio de mercado brasileiro; não obstante, deve-se ter em vista que são estimativas ex post, sujeitas, portanto, às críticas já reportadas no item Revisão Bibliográfica. 
Vale lembrar também a limitação dessas estimações quanto aos erros envolvidos, mesmo sendo uma amostra representativa (156 retornos ou observações mensais, ao longo de 13 anos consecutivos, ou ainda 3.219 dias de negociação); trata-se de retornos de frequência mais alta que o ideal para a finalidade em tela; portanto são resultados sujeitos às flutuações (e dispersão) mais intensas do curto prazo, razão por que, isoladamente, é suficiente para nos levar ao próximo método para estimação.

Assim sendo, conforme descrito em Abordagem Prospectiva ou Implícita, foram obtidos, para cada uma das 131 empresas listadas nesse mesmo item, o dividend yield médio, o crescimento médio, a partir da média das taxas de reinvestimento de cada empresa; estas dependem, por sua vez, da retenção dos lucros e do ROE em cada período e a taxa média de retorno esperada, que é a soma de ambas as estimativas anteriores, conforme explicitado por (1); conforme demonstra a Tabela A-1 (vide Apêndice) e está predisposto em (2), as médias simples e ponderada pelos respectivos patrimônios líquidos das empresas (incluindo ou não aquelas consideradas como outliers) seguem na Tabela 6.

Tabela 6

Estimativas Ex ante do Retorno da Carteira de Mercado

\begin{tabular}{ccc}
\hline $\mathbf{E}\left(\mathbf{R}_{\mathbf{m})}\right.$ & com outliers & sem outliers \\
\hline Média simples & $17,838 \%$ & $17,314 \%$ \\
Média ponderada & $7,748 \%$ & $11,989 \%$ \\
\hline
\end{tabular}

Se considerarmos o valor simples sem os outliers como o mais confiável, tendo em vista que duas das empresas da amostra - PETR4 e VALE5 - influenciam decisivamente os resultados por conta de seu tamanho, da retenção de lucros e, consequentemente, da taxa de crescimento - em particular a última, considerada outlier; e tomarmos a média ponderada (pelo número de contratos negociados) dos retornos nominais esperados (ao ano, base 252 dias úteis) dos contratos DI 1 dia para vencimento em janeiro de 2017 (maturidade mais longínqua disponível) abertos em dezembro de 2008 (fim da janela histórica de trabalho desta abordagem) vemos que a taxa livre de risco média a ser considerada é $13,965 \%$, o que nos leva a um prêmio de risco esperado de $\mathbf{3 , 3 5 0 \%}$.

Resta assim apresentar os resultados encontrados na terceira e última abordagem, a indireta. A populosa amostra utilizada nas inferências resultou em dois painéis desbalanceados (regressão com variáveis em nível e em diferenças), respectivamente com 462 e 8.293 observações.

Note-se, como demonstram os resultados da Tabela 7, que as séries de $\beta^{\mathrm{i}}$ em nível podem ser não estacionárias no período. Testes conduzidos individualmente para todos os ativos mostraram, entretanto, que EMBR3, SBSP3, TBLE3 e TRPL4 são estacionárias nos $\beta^{i}$ e nos respectivos retornos excedentes (prêmios); isso permitiria a condução de uma regressão em painel com variáveis em nível para esses quatro ativos, o que não impede, porém, a recondução de um painel em diferenças, empregando todos os ativos, como proposto em (4).

Tabela 7

Principais Estatísticas Descritivas da Amostra com Testes de Estacionariedade

\begin{tabular}{lcccccccc}
\hline & \multicolumn{3}{c}{ Retornos Contínuos } & \multicolumn{3}{c}{ Retornos Discretos } \\
\cline { 2 - 9 } & \multicolumn{2}{c}{ Betas } & \multicolumn{2}{c}{ Prêmios } & \multicolumn{2}{c}{ Betas } & \multicolumn{2}{c}{ Prêmios } \\
\cline { 2 - 9 } & Em nível & $\mathbf{1}^{\text {a diferença }}$ & Em nível & $\mathbf{1}^{\text {a }}$ diferença & Em nível & $\mathbf{1}^{\text {a }}$ diferença & Em nível & $\mathbf{1}^{\text {a }}$ diferença \\
\hline Média & 0,6529 & 0,002043 & $0,003 \%$ & $-0,002 \%$ & 0,6530 & 0,001965 & $0,003 \%$ & $-0,002 \%$ \\
Mediana & 0,6181 & 0,000960 & $0,015 \%$ & $-0,002 \%$ & 0,6175 & 0,000948 & $0,015 \%$ & $-0,002 \%$ \\
\hline
\end{tabular}


Tabela 7 (continuação)

\begin{tabular}{|c|c|c|c|c|c|c|c|c|}
\hline & \multicolumn{4}{|c|}{ Retornos Contínuos } & \multicolumn{4}{|c|}{ Retornos Discretos } \\
\hline & \multicolumn{2}{|c|}{ Betas } & \multicolumn{2}{|c|}{ Prêmios } & \multicolumn{2}{|c|}{ Betas } & \multicolumn{2}{|c|}{ Prêmios } \\
\hline & Em nível & $1^{a}$ diferença & Em nível & $1^{\text {a }}$ diferença & Em nível & $1^{\text {a }}$ diferença & Em nível & $1^{a}$ diferença \\
\hline Máximo & 3,6247 & 11,9391 & $1,033 \%$ & $0,546 \%$ & 3,6971 & 11,7561 & $1,039 \%$ & $0,547 \%$ \\
\hline Mínimo & $-9,6678$ & $-2,2904$ & $-1,395 \%$ & $-0,659 \%$ & $-9,4652$ & $-2,2862$ & $-1,386 \%$ & $-0,660 \%$ \\
\hline Desvio padrão & 0,3412 & 0,1674 & $0,209 \%$ & $0,077 \%$ & 0,3395 & 0,1652 & $0,209 \%$ & $0,077 \%$ \\
\hline Assimetria & $-3,604$ & 62,456 & $-0,422$ & 0,052 & $-3,387$ & 62,026 & $-0,410$ & 0,052 \\
\hline Curtose & 112,89 & $4.302,47$ & 4,93 & 5,76 & 105,69 & $4.266,57$ & 4,91 & 5,75 \\
\hline Jarque-Bera & $0,00 \%$ & $0,00 \%$ & $0,00 \%$ & $0,00 \%$ & $0,00 \%$ & $0,00 \%$ & $0,00 \%$ & $0,00 \%$ \\
\hline \multirow{2}{*}{$\begin{array}{l}\text { Im, Pesaran e } \\
\text { Shin W-estat. }\end{array}$} & 4,7313 & $-46,5061$ & $-6,0641$ & $-42,7604$ & 4,9457 & $-46,6084$ & $-6,0778$ & $-42,7560$ \\
\hline & $100,00 \%$ & $0,00 \%$ & $0,00 \%$ & $0,00 \%$ & $100,00 \%$ & $0,00 \%$ & $0,00 \%$ & $0,00 \%$ \\
\hline \multirow{2}{*}{$\begin{array}{l}\text { ADF - Fisher } \\
\text { Chi-quadrado }\end{array}$} & 148,40 & $2.222,59$ & 278,46 & $2.092,88$ & 145,75 & $2.216,88$ & 278,81 & $2.092,61$ \\
\hline & $65,52 \%$ & $0,00 \%$ & $0,00 \%$ & $0,00 \%$ & $71,08 \%$ & $0,00 \%$ & $0,00 \%$ & $0,00 \%$ \\
\hline \multirow{2}{*}{$\begin{array}{l}\text { PP - Fisher } \\
\text { Chi-quadrado }\end{array}$} & 491,17 & $4.792,45$ & 365,09 & $4.676,94$ & 494,78 & $4.803,45$ & 365,61 & $4.677,54$ \\
\hline & $0,00 \%$ & $0,00 \%$ & $0,00 \%$ & $0,00 \%$ & $0,00 \%$ & $0,00 \%$ & $0,00 \%$ & $0,00 \%$ \\
\hline Observações & 10.114 & 10.036 & 9.692 & 9.158 & 10.114 & 10.036 & 9.692 & 9.158 \\
\hline
\end{tabular}

Nota. Fonte: Elaboração própria.

Tabela 8

Resultados Obtidos no Painel Conduzido Segundo o Especificado em (3) e (4)

\begin{tabular}{|c|c|c|c|c|c|c|c|c|}
\hline & \multicolumn{8}{|c|}{ Retornos Discretos } \\
\hline & \multicolumn{4}{|c|}{ Em nível } & \multicolumn{4}{|c|}{$1^{a}$ Diferença } \\
\hline & \multicolumn{2}{|c|}{ Efeitos Aleatórios } & \multicolumn{2}{|c|}{ Efeitos Fixos } & \multicolumn{2}{|c|}{ Efeitos Aleatórios } & \multicolumn{2}{|c|}{ Efeitos Fixos } \\
\hline & Coeficiente & P-Valor & Coeficiente & P-Valor & Coeficiente & P-Valor & Coeficiente & P-Valor \\
\hline Intercepto & $0,1533 \%$ & $0,000 \%$ & $0,1358 \%$ & $0,419 \%$ & $-0,0024 \%$ & $54,124 \%$ & $-0,0026 \%$ & $0,021 \%$ \\
\hline Prêmio de Mercado & $-0,1724 \%$ & $3,349 \%$ & $-0,1357 \%$ & $9,983 \%$ & $-0,0878 \%$ & $2,132 \%$ & $-0,0904 \%$ & $1,702 \%$ \\
\hline $\mathbf{R}^{2}$ ajustado & \multicolumn{2}{|c|}{0,0523} & \multicolumn{2}{|c|}{0,4490} & \multicolumn{2}{|c|}{0,0008} & \multicolumn{2}{|c|}{0,3323} \\
\hline Durbin-Watson & \multicolumn{2}{|c|}{0,1719} & \multicolumn{2}{|c|}{0,2202} & \multicolumn{2}{|c|}{1,9896} & \multicolumn{2}{|c|}{1,9946} \\
\hline Períodos incluídos & \multicolumn{4}{|c|}{134} & \multicolumn{4}{|c|}{134} \\
\hline Ativos incluídos & \multicolumn{4}{|c|}{4} & \multicolumn{4}{|c|}{78} \\
\hline Observações & \multicolumn{4}{|c|}{462} & \multicolumn{4}{|c|}{8.293} \\
\hline Hausman & \multicolumn{4}{|c|}{20,973} & \multicolumn{4}{|c|}{2,990} \\
\hline P-Valor Hausman & \multicolumn{4}{|c|}{$0,000 \%$} & \multicolumn{4}{|c|}{$8,381 \%$} \\
\hline
\end{tabular}

\begin{tabular}{|c|c|c|c|c|c|c|c|c|}
\hline & \multicolumn{8}{|c|}{ Retornos Contínuos } \\
\hline & \multicolumn{4}{|c|}{ Em nível } & \multicolumn{4}{|c|}{$1^{\mathrm{a}}$ Diferença } \\
\hline & \multicolumn{2}{|c|}{ Efeitos Aleatórios } & \multicolumn{2}{|c|}{ Efeitos Fixos } & \multicolumn{2}{|c|}{ Efeitos Aleatórios } & \multicolumn{2}{|c|}{ Efeitos Fixos } \\
\hline & Coeficiente & P-Valor & Coeficiente & P-Valor & Coeficiente & P-Valor & Coeficiente & P-Valor \\
\hline Intercepto & $0,1555 \%$ & $0,000 \%$ & $0,1380 \%$ & $0,450 \%$ & $-0,0024 \%$ & $54,161 \%$ & $-0,0026 \%$ & $0,021 \%$ \\
\hline Prêmio de Mercado & $-0,1755 \%$ & $2,511 \%$ & $-0,1389 \%$ & $9,248 \%$ & $-0,0820 \%$ & $3,427 \%$ & $-0,0842 \%$ & $2,878 \%$ \\
\hline $\mathbf{R}^{2}$ ajustado & \multicolumn{2}{|c|}{0,0524} & \multicolumn{2}{|c|}{0,4501} & \multicolumn{2}{|c|}{0,0006} & \multicolumn{2}{|c|}{0,3323} \\
\hline
\end{tabular}




\section{Tabela 8 (continuação)}

\begin{tabular}{|c|c|c|c|c|c|c|c|c|}
\hline & \multicolumn{8}{|c|}{ Retornos Contínuos } \\
\hline & \multicolumn{4}{|c|}{ Em nível } & \multicolumn{4}{|c|}{$1^{\text {a }}$ Diferença } \\
\hline & \multicolumn{2}{|c|}{ Efeitos Aleatórios } & \multicolumn{2}{|c|}{ Efeitos Fixos } & \multicolumn{2}{|c|}{ Efeitos Aleatórios } & \multicolumn{2}{|c|}{ Efeitos Fixos } \\
\hline & Coeficiente & P-Valor & Coeficiente & P-Valor & Coeficiente & P-Valor & Coeficiente & P-Valor \\
\hline Durbin-Watson & 0,17 & & 0,22 & & 1,98 & & 1,99 & \\
\hline Períodos incluídos & \multicolumn{4}{|c|}{134} & \multicolumn{4}{|c|}{134} \\
\hline Ativos incluídos & \multicolumn{4}{|c|}{4} & \multicolumn{4}{|c|}{78} \\
\hline Observações & \multicolumn{4}{|c|}{462} & \multicolumn{4}{|c|}{8.293} \\
\hline Hausman & \multicolumn{4}{|c|}{20,261} & \multicolumn{4}{|c|}{2,248} \\
\hline P-Valor Hausman & \multicolumn{4}{|c|}{$0,001 \%$} & \multicolumn{4}{|c|}{$13,375 \%$} \\
\hline
\end{tabular}

Nota. Regressões em nível empregaram painel com somente os ativos EMBR3, SBSP3, TBLE3 E TRPL4. As regressões em $1^{\text {a }}$ diferença empregaram todos os ativos relacionados na Tabela 3 . Todas as regressões conduzidas pelo método GMM, erros robustos segundo White (1980), supondo-se autocorrelação e heterocedasticidade nas séries temporais de cada ativo (método White-period). Considerados efeitos fixos ou aleatórios temporais ao intercepto. A frequência dos dados é mensal; os Betas foram estimados em janela móvel de abrangência trianual; os retornos, similarmente, são cumulativos anuais.

Fonte. Elaboração própria.

Assim, um primeiro resultado a se destacar na Tabela 8 são as estatísticas de Durbin-Watson que, como já previsto, dão conta da alta persistência dos betas em nível, algo que as diferenças mitigam sobremaneira; é notável também que, embora o prêmio de mercado estimado varie consideravelmente de acordo com o método e tipo de retorno empregado, ele é inequivocamente negativo e significativo, variando em termos anualizados de $-19 \%$ a $-35 \%$. Trata-se, portanto, de resultado robusto, mas que surpreende pelo contraste com as estimativas anteriores e o senso comum. Vale comentar ainda que o modelo de painel com efeito fixo é o que é validado pelo Teste de Hausman, algo que os $R^{2}$ ajustados (o indicador de aderência disponível na saída oferecida pelo EViews-7® para este tipo de regressão) também parecem sugerir como a melhor abordagem econométrica para o caso.

\section{Considerações Finais}

$\mathrm{O}$ artigo mostrou, conseguintemente, estimativas ex post para o prêmio de mercado bastante similares às encontradas pela literatura acadêmica; ficou registrado também algo já documentado em diversos trabalhos a respeito das menores taxas para o prêmio de mercado esperado em relação aos valores historicamente observados, assim como um resultado inusitado segundo a abordagem econométrica, valendo-se para tanto do previsto pelo CAPM na Securities Market Line (SML).

Cabem, no entanto, alguns comentários acerca desses resultados encontrados. Com relação às estimativas ex post, Campbell (2007) emprega o uso de médias móveis de três anos para as estimativas, como forma de mitigar os efeitos decorrentes da volatilidade das séries usadas, algo não considerado neste artigo senão na produção de uma figura mais pictórica, dada a autocorrelação que inevitavelmente surge nas estimativas; porém trata-se de uma abordagem possível, que certamente produziria resultados diferentes dos aqui apresentados.

Quanto à janela histórica que serviu de base para o presente estudo nas três abordagens, embora embasada em conveniências ligadas às idiossincrasias dos índices brasileiros e ao histórico econômico do país, de forma a evitar a importante quebra estrutural inequivocamente presente a essas séries que é o Plano Real (julho de 1994), ela indubitavelmente também exerce considerável influência. Benartzi e Thaler (1995) alertam para o efeito que o horizonte de investimento exerce nas estimativas, função da 
aversão ao risco e da volatilidade do mercado; juros reais e inflação também são importantes influenciadores nesse sentido, conforme atestado em Blanchard (1993).

Outro aspecto relevante a ser destacado é a inconstância temporal do prêmio de mercado, tendo em vista a possível, porém estreita, janela definida como objeto de estudo: é inevitável a alusão da hipótese de que tais valores são estritamente ligados ao período em questão, não representando, portanto, um componente permanente da média verdadeira. Nesse caso, resultados como os da $3^{\text {a }}$ abordagem não deveriam surpreender; haja vista que o período em questão foi marcado por alguns cenários adversos importantes, intercalados por períodos de estagnação e otimismo, como expresso anteriormente na Figura 1; isto daria suporte, em alguma medida, às idéias originalmente preconizadas por Rietz (1988), embora sobre eles não possamos afirmar categoricamente que se trata de rupturas; fato é, em verdade, que para essa questão não há respostas prontas e os dados ora disponíveis são, provavelmente, insuficientes, terreno fértil, sem dúvida, para contribuições futuras.

Tais dificuldades não devem, no entanto, representar óbice para aprimoramentos metodológicos e procedimentais, em busca de resultados mais refinados: a abordagem 2, por exemplo, parte de modo simplista do pressuposto de que todas as empresas incluídas na estimativa se encontram em estágio maduro em sua geração de resultados, algo que poderia ser individualmente apreciado, visando à aplicação do modelo de dois ou mais estágios; outros estimadores relevantes do valor para a avaliação em perpetuidade (como FCFF), ou ainda proxies mais explicativas para o crescimento das empresas poderiam, em tese, levar a resultados mais refinados; estas empreitas, pela laboriosidade envolvida na apropriação e tratamento dos dados, justificariam um trabalho especificamente voltado a esse objeto.

A terceira abordagem, se cumprido um esforço equivalente, também poderia trazer melhores subsídios, mediante diferentes métodos econométricos e variáveis instrumentais, visando a maior robustez do tratamento estatístico; por fim, mas não menos importante, uma análise quantitativa de sensibilidade para outliers e/ou observações influentes, que são as possíveis responsáveis pelos inquietantes resultados ora demonstrados.

Artigo recebido em 26.03.2010. Aprovado em 24.05.2011.

\section{Referências}

Abel, B. A. (1990). Asset prices under habit formation and catching up with the Joneses. American Economic Review, Papers and Proceedings, 80(2), 38-42.

Aiyagari, S. K., \& Gertler, M. (1991). Asset returns with transactions costs and uninsured individual risk. Journal of Monetary Economics, 27(3), 311-331. doi: 10.1016/0304-3932(91)90012-D

Alencar, A. S. (2002). Testando CAPM através das fronteiras de volatilidade e da equação de Euler. In M. Bonomo (Ed.), Finanças aplicadas ao Brasil (pp. 119-161). Rio de Janeiro: Editora da Fundação Getulio Vargas.

Alpalhão, R., \& Alves, P. (2005). The Portuguese equity risk premium: what we know and what we don't know. Applied Financial Economics, 15(7), 489-498. doi: 10.1080/09603100500038799

Alvarez, F., \& Jermann, U. (2000). Efficiency, equilibrium, and asset pricing with risk of default. Econometrica, 68(4), 775-797. doi: 10.1111/1468-0262.00137

Araújo, A. M. P., Assaf, A., Neto, \& Lima, F. (2006, julho). Metodologia de cálculo do custo de capital no Brasil. Anais do Congresso USP de Contabilidade, São Paulo, SP, Brasil, 6. 
Araújo, E. A., Jr. (2005). Avaliando três especificações para o fator de desconto estocástico através da fronteira de volatilidade de Hansen-Jaganathan: um estudo empírico para o Brasil. Pesquisa e Planejamento Econômico, 35(1), 49-73.

Arnott, R., \& Bernstein, P. (2002). What risk premium is normal? Financial Analysts Journal, 58(2), 64-85. doi: 10.2469/faj.v58.n2.2524

Bansal, R., \& Coleman, J. W. (1996). A monetary explanation of the equity premium, term premium, and risk-free rate puzzles. Journal of Political Economy, 104(6), 1135-1171.

Benartzi, S., \& Thaler, R. H. (1995). Myopic loss aversion and the equity premium puzzle. Quarterly Journal of Economics, 110(1), 73-92. doi: 10.2307/2118511

Blanchard, O. J. (1993). Movements in the equity premium. Brookings Papers on Economic Activity, Macroeconomics, (2), 75-118.

Boldrin, M., Christiano, J. L., \& Fisher, J. D. M. (2001). Habit persistence, asset returns, and the business cycle. The American Economic Review, 91(1), 149-166. doi: 10.1257/aer.91.1.149

Bonomo, M., \& Domingues, G. (2002). Os puzzles invertidos no mercado brasileiro de ativos. In M. Bonomo (Ed.), Finanças aplicadas ao Brasil (pp. 105-120). Rio de Janeiro: FGV Editora.

Bonomo, M., \& Garcia, R. (1994). Disappointment aversion as a solution to the equity premium and the risk-free rate puzzles [Working Paper $\mathrm{N}^{\circ}$ 94s-14]. Centre Interuniversitaire de Recherche en Analyse des Organisations, Montréal, Canada.

Brown, S., Goetzmann, W., \& Ross, S. (1995). Survival. Journal of Finance, 50(2), 853-873.

Campbell, J. Y. (2007). Estimating the equity premium [Working Paper $\mathrm{N}^{\circ}$ 13423]. National Bureau of Economic Research, Cambridge, MA.

Campbell, J. Y., \& Cochrane, J. H. (1999). By force of habit: a consumption based explanation of aggregate stock market behavior. Journal of Political Economy, 107(2), 205-251. doi: $10.1086 / 250059$

Canova, F., \& Nicoló, G. (2003). The properties of the equity premium and the risk-free rate: an investigation across time and countries. IMF Staff Papers, 50(2), 222-249.

Catalão, A. B., \& Yoshino, J. A. (2006). Fator de desconto estocástico no mercado acionário brasileiro. Estudos Econômicos, 36(3), 435-463. doi: 10.1590/S0101-41612006000300002

Ceccheti, S. G., \& Mark, N. C. (1990). Evaluating empirical tests of asset pricing models: alternative interpretations. American Economic Review, 80(2), 48-51.

Claus, J., \& Thomas, J. (2001). Equity premia as low as three percent? Evidence from analysts' earnings forecasts for domestic and international stock markets. Journal of Finance, 56(5), 1629-1666. doi: 10.1111/0022-1082.00384

Constantinides, G. M. (1990). Habit formation: a resolution of the equity premium puzzle. Journal of Political Economy, 98(3), 519-543. doi: 10.1086/261693

Constantinides, G. M., Donaldson, J. B., \& Mehra, R. (2002). Junior can't borrow: a new perspective on the equity premium puzzle. Quarterly Journal of Economics, 117(1), 269-296. doi: $10.2139 /$ ssrn. 206568

Constantinides, G. M., \& Duffie, D. (1996). Asset pricing with heterogeneous consumers. Journal of Political Economy, 104(2), 219-240. doi: 10.1086/262023 
Cornell, B. (1999). The equity risk premium: the long-run future of the stock market. New York: John Wiley \& Sons.

Corrêa, L. S., \& Samanez, C. P. (2002). Análise e avaliação do prêmio de risco nos mercados acionários brasileiro e americano (Dissertação de mestrado). Pontifícia Universidade Católica, Rio de Janeiro, RJ, Brasil.

Cysne, R. P. (2005). Equity-premium puzzle: evidence from Brazilian data [Working Paper No 586]. Fundação Getúlio Vargas, Rio de Janeiro, Brasil.

Damodaran, A. (2001). Estimating equity risk premiums [Working Paper]. Stern Scholl of Business, New York, USA.

Danthine, J. P., \& Donaldson, J. B. (1999). Non-falsified expectations and general equilibrium asset pricing: the power of the peso. The Economic Journal, 109(458), 607-635. doi: 10.1111/14680297.00465

Dimson, E., Marsh, P., \& Staunton, M. (2003). Global evidence on the equity risk premium. Journal of Applied Corporate Finance, 15(4), 27-38. doi: 10.1111/j.1745-6622.2003.tb00524.x

Epstein, L. G., \& Zin, S. E. (1991). Substitution, risk aversion, and the temporal behavior of consumption growth and asset returns II: an empirical analysis. Journal of Political Economy, 99(2), 263-287.

Fama, E. F., \& French, K. R. (2002). The equity premium. Journal of Finance, 57(2), 637-659. doi: $10.1111 / 1540-6261.00437$

Ferson, W. E., \& Constantinides, G. (1991). Habit persistence and durability in aggregate consumption: empirical tests. Journal of Financial Economics, 29(2), 199-240. doi: $10.1016 / 0304-405 X(91) 90002-2$

Freeman, M. C., \& Davidson, I. R. (1999). Estimating the equity premium. The European Journal of Finance, 5(3), 236-246. doi: 10.1080/135184799337073

Gordon, J. M. (1962). The investment, financing, and valuation of the corporation. Irwin: Homewood.

Goulart, A. M. C, \& Paiva, E. V. S. (2005, outubro). Prêmio de risco no mercado de renda variável brasileiro (1986 a 2004). Anais do Congresso USP de Contabilidade, são Paulo, SP, Brasil, 5.

Guvenen, F. A. (2005). Parsimonious macroeconomic model for asset pricing: habit formation or cross-sectional heterogeneity? [Working Paper $\mathrm{N}^{\circ}$ 499]. University of Rochester, Rochester, New York, USA.

Hansen, P., Sargent, T., \& Tallarini, T. (1994). Pessimism, neurosis and feelings about risk in general equilibrium. [Working Paper]. University of Chicago, Chicago, USA.

Heaton, J., \& Lucas, D. (1996). Evaluating the effects of incomplete markets on risk sharing and asset pricing. Journal of Political Economy, 104(3), 443-487. doi: 10.2307/2138860

Heaton, J., \& Lucas, D. (1997). Market frictions, savings behavior and portfolio choice. Journal of Microeconomic Dynamics, 1(1), 76-101. doi: 10.1017/S1365100597002034

Ibbotson Associates. (1992). Stocks, bonds, bills and inflation. Chicago: Author.

Jagannathan, R., McGrattan, R. E., \& Scherbina, A. (2000). The declining U.S. equity premium. Quarterly Review Federal Reserve Bank of Minneapolis, 24(4), 3-19.

Jermann, J. U. (1998). Asset pricing in production economies. Journal of Monetary Economics, 41(2), 257-275. doi: 10.1016/S0304-3932(97)00078-0 
Jorion, P., \& Goetzmann, W. N. (1999). Global stock markets in the twentieth century. Journal of Finance, 54(3), 953-980. doi: 10.1111/0022-1082.00133

Kocherlakota, N. R. (1996). The equity premium: it's still a puzzle. Journal of Economic Literature, 34(1), 42-71.

Leal, R. P. C. (2002). Revisão da literatura sobre estimativa de custo de capital aplicada ao Brasil. Rio de Janeiro: Coppead/UFRJ.

Lungu, L., \& Minford, P. (2006). Explaining the equity risk premium. Manchester School, 74(6), 670700. doi: $10.1111 /$ j.1467-9957.2006.00522.x

Mankiw, N. G. (1986). The equity premium and the concentration of aggregate shocks. Journal of Financial Economics, 17(1), 211-219. doi: 10.1016/0304-405X(86)90012-7

McGrattan, E. R., \& Prescott, E. C. (2005). Taxes, regulations and the value of U.S. and U.K. corporations. Review of Economic Studies, 72(3), 767-796. doi: 10.1111/j.1467937X.2005.00351.X

Mehra, R. (2003). The equity premium: why is it a puzzle? Financial Analysts Journal, 59(1), 54-69. doi: 10.2469/faj.v59.n1.2503

Mehra, R., \& Prescott, E. C. (1985). The equity premium: a puzzle. Journal of Monetary Economics, 15(2), 145-161. doi: 10.1016/0304-3932(85)90061-3

Miller, M., Weller, P., \& Zhang, L. (2002). Moral hazard and the US stock market: analyzing the "Greenspan Put". The Economic Journal, 112(478), C171-C186. doi: 10.1111/1468-0297.00029

Minardi, A. M. A. F., \& Sanvicente, A. Z. (2006, julho). Análise da série histórica de prêmios pelo risco de mercado estimados pelo modelo de dividendos descontados. Anais do Encontro Brasileiro de Finanças, Vitória, ES, Brasil, 6.

Pastor, L., Sinha, M., \& Swaminathan, B. (2008). Estimating the intertemporal risk-return tradeoff using the implied cost of capital. Journal of Finance, 63(6), 2859-2897. doi: 10.1111/j.15406261.2008.01415.x

Pastor, L., \& Stambaugh, R. (2001). The equity premium and structural breaks. Journal of Finance, 56(4), 1207-1239. doi: 10.1111/0022-1082.00365

Rietz, T. (1988). The equity risk premium a solution. Journal of Monetary Economics, 22(1), 117-131. doi: 10.1016/0304-3932(88)90172-9

Salomons, R., \& Grootveld, H. (2003). The equity risk premium: emerging vs. developed markets. Emerging Markets Review, 4(2), 121-144. doi: 10.1016/S1566-0141(03)00024-4

Sampaio, F. S. (2002). Existe equity premium puzzle no Brasil? In M. Bonomo (Org.), Finanças aplicadas ao Brasil (pp. 8-25). Rio de Janeiro: Editora FGV.

Santos, R. C. (2006). Análise e avaliação do equity premium puzzle no mercado acionário brasileiro sob diferentes contextos econômicos (Dissertação de mestrado). Pontifícia Universidade Católica, Rio de Janeiro, RJ, Brasil.

Schöeder, D. (2007). The implied equity risk premium - an evaluation of empirical methods. Kredit und Kapital, 40(4), 583-613.

Siegel, J. (1992). The equity premium: stocks and bond returns since 1802. Financial Analysts Journal, 48(1), 28-38. doi: 10.2469/faj.v48.n1.28 
Soriano, A. (2002). Testando o CAPM através das fronteiras de volatilidade e da equação de Euler. In M. Bonomo (Org.), Finanças aplicadas ao Brasil (pp. 8-25). Rio de Janeiro: Editora FGV.

Storesletten, K., Telmer, C. I., \& Yaron, A. (2007). Asset pricing with idiosyncratic risk and overlapping generations. Review of Economic Dynamics, 10(4), 519-548. doi: 10.1016/j.red.2007.02.004

Sundersan, S. M. (1989). Intertemporally dependent preferences and the volatility of consumption and wealth. Review of Financial Studies, 2(1), 73-88. doi: 10.1093/rfs/2.1.73

Weil, P. (1992). Equilibrium asset prices with undiversifiable labour income risk. Journal of Economic Dynamics and Control, 16(3-4), 769-790. doi: 10.1016/0165-1889(92)90057-L

White, H. (1980). A heteroskedasticity consistent covariance matrix estimator and a direct test of heteroskedasticity. Econometrica, 58(4), 817-838. 


\section{Apêndice}

Tabela A-1

\section{Resultados Parciais para a Abordagem Prospectiva Segundo a Equação (1)}

\begin{tabular}{|c|c|c|c|c|c|c|c|c|c|c|c|}
\hline Nome de Pregão & Código & Fim (período) & $\mathbf{D}_{1} / \mathbf{P}_{0}$ & G & $\mathbf{R}_{\mathbf{i}}$ & Nome de Pregão & Código & Fim (período) & $\mathbf{D}_{1} / \mathbf{P}_{0}$ & G & $\mathbf{R}_{\mathbf{i}}$ \\
\hline ABNOTE & ABNB3 & $17 / 12 / 2007$ & $2,5398 \%$ & $28,6038 \%$ & $31,1436 \%$ & TREVISA & LUXM4 & $26 / 3 / 2007$ & $3,7193 \%$ & $59,9999 \%$ & $63,7192 \%$ \\
\hline ALL AMER LAT & ALLL4 & $28 / 4 / 2008$ & $0,3910 \%$ & $4,3681 \%$ & $4,7591 \%$ & JEREISSATI & MFLT4 & $28 / 4 / 2008$ & $3,3819 \%$ & $9,1859 \%$ & $12,5678 \%$ \\
\hline ALPARGATAS & ALPA4 & $22 / 11 / 2007$ & $6,0279 \%$ & $15,8390 \%$ & $21,8669 \%$ & MANGELS INDL & MGEL4 & $27 / 4 / 2007$ & $2,6250 \%$ & $2,1730 \%$ & $4,7980 \%$ \\
\hline AMBEV & AMBV4 & $10 / 12 / 2007$ & $8,5195 \%$ & $18,2153 \%$ & $26,7348 \%$ & MONT ARANHA & MOAR3 & $17 / 1 / 2008$ & $13,5505 \%$ & $15,3708 \%$ & $28,9213 \%$ \\
\hline ARACRUZ & ARCZ6 & $25 / 9 / 2007$ & $3,5148 \%$ & $17,6892 \%$ & $21,2039 \%$ & MARISOL & MRSL4 & $19 / 2 / 2008$ & $5,0557 \%$ & $12,6822 \%$ & $17,7380 \%$ \\
\hline ACOS VILL & AVIL3 & $13 / 11 / 2008$ & $4,0361 \%$ & $44,1469 \%$ & $48,1830 \%$ & METISA & MTSA4 & $20 / 12 / 2007$ & $7,9592 \%$ & $17,4948 \%$ & $25,4540 \%$ \\
\hline BARDELLA & BDLL4 & $21 / 12 / 2006$ & $11,5425 \%$ & $1,2574 \%$ & $12,7999 \%$ & NADIR FIGUEI & NAFG4 & $28 / 2 / 2005$ & $3,6095 \%$ & $0,0000 \%$ & $3,6095 \%$ \\
\hline BRASMOTOR & BMTO4 & $18 / 1 / 2008$ & $5,5594 \%$ & $7,7694 \%$ & $13,3288 \%$ & NATURA & NATU3 & $30 / 7 / 2007$ & $3,7751 \%$ & $-28,138 \%$ & $-24,3630 \%$ \\
\hline EST PIAUI & BPIA3 & $8 / 12 / 2008$ & $2,8825 \%$ & $16,1211 \%$ & $19,0036 \%$ & SUZANO HOLD & NEMO4 & $19 / 4 / 2005$ & $0,8121 \%$ & $17,9037 \%$ & $18,7158 \%$ \\
\hline BRADESPAR & BRAP4 & $31 / 10 / 2007$ & $3,4882 \%$ & $2,1096 \%$ & $5,5978 \%$ & PAUL F LUZ & PALF3 & $19 / 8 / 2005$ & $5,9212 \%$ & $9,2898 \%$ & $15,2110 \%$ \\
\hline BRASKEM & BRKM5 & $28 / 3 / 2007$ & $7,5765 \%$ & $6,0097 \%$ & $13,5862 \%$ & PANATLANTICA & PATI4 & $11 / 4 / 2007$ & $16,6745 \%$ & $25,3133 \%$ & $41,9878 \%$ \\
\hline BRASIL TELEC & BRTO4 & $26 / 12 / 2007$ & $5,7760 \%$ & $5,8205 \%$ & $11,5966 \%$ & P.ACUCAR-CBD & PCAR4 & $30 / 4 / 2007$ & $1,3848 \%$ & $8,4164 \%$ & $9,8012 \%$ \\
\hline BRASIL T PAR & BRTP4 & $26 / 12 / 2007$ & $5,0608 \%$ & $5,7579 \%$ & $10,8186 \%$ & PAR AL BAHIA & PEAB4 & $12 / 12 / 2007$ & $6,0105 \%$ & $8,5631 \%$ & $14,5736 \%$ \\
\hline CCR RODOVIAS & CCRO3 & $9 / 8 / 2007$ & $4,8979 \%$ & $-0,2887 \%$ & $4,6092 \%$ & PETROBRAS & PETR4 & $11 / 1 / 2008$ & $4,3584 \%$ & $-7,4076 \%$ & $-3,0492 \%$ \\
\hline COELBA & CEEB3 & $17 / 12 / 2007$ & $13,8566 \%$ & $25,8349 \%$ & $39,6914 \%$ & DIMED & PNVL3 & $14 / 12 / 2007$ & $4,9172 \%$ & $9,5712 \%$ & $14,4884 \%$ \\
\hline CELPE & CEPE5 & $19 / 12 / 2007$ & $12,0473 \%$ & $11,4162 \%$ & $23,4635 \%$ & MARCOPOLO & POMO4 & $14 / 12 / 2007$ & $7,1587 \%$ & $4,7922 \%$ & $11,9509 \%$ \\
\hline COMGAS & CGAS5 & $16 / 10 / 2007$ & $5,7147 \%$ & $27,3927 \%$ & $33,1075 \%$ & PETROQ UNIAO & PQUN3 & $28 / 12 / 2007$ & $8,9647 \%$ & $14,0911 \%$ & $23,0558 \%$ \\
\hline GRAZZIOTIN & CGRA4 & $26 / 12 / 2007$ & $7,8710 \%$ & $12,8179 \%$ & $20,6889 \%$ & PERDIGAO S/A & PRGA3 & $28 / 12 / 2007$ & $3,5138 \%$ & $7,9196 \%$ & $11,4335 \%$ \\
\hline CELESC & CLSC3 & $21 / 11 / 2007$ & $2,5213 \%$ & $13,3612 \%$ & $15,8825 \%$ & PORTO SEGURO & PSSA3 & $30 / 12 / 2008$ & $4,1907 \%$ & $4,8935 \%$ & $9,0841 \%$ \\
\hline CEMIG & CMIG4 & $11 / 7 / 2006$ & $6,0025 \%$ & $11,8649 \%$ & $17,8675 \%$ & PETTENATI & PTNT4 & $25 / 10 / 2007$ & $6,7910 \%$ & $8,2720 \%$ & $15,0630 \%$ \\
\hline
\end{tabular}


Tabela A-1 (continuação)

\begin{tabular}{|c|c|c|c|c|c|c|c|c|c|c|c|}
\hline Nome de Pregão & Código & Fim (período) & $\mathbf{D}_{1} / \mathbf{P}_{0}$ & G & $\mathbf{R}_{\mathbf{i}}$ & Nome de Pregão & Código & Fim (período) & $\mathbf{D}_{1} / \mathbf{P}_{\mathbf{0}}$ & G & $\mathbf{R}_{\mathbf{i}}$ \\
\hline CONFAB & CNFB4 & $30 / 10 / 2007$ & $8,2851 \%$ & $8,6146 \%$ & $16,8997 \%$ & PETROPAR & PTPA4 & $19 / 11 / 2007$ & $13,0426 \%$ & $13,2928 \%$ & $26,3353 \%$ \\
\hline COELCE & COCE5 & $30 / 4 / 2007$ & $8,0704 \%$ & $9,5933 \%$ & $17,6637 \%$ & RANDON PART & RAPT4 & $3 / 12 / 2007$ & $3,0759 \%$ & $23,7843 \%$ & $26,8602 \%$ \\
\hline CPFL ENERGIA & CPFE3 & $14 / 8 / 2007$ & $9,1776 \%$ & $7,6108 \%$ & $16,7884 \%$ & RECRUSUL & RCSL4 & $16 / 4 / 2004$ & $5,1347 \%$ & $8,2221 \%$ & $13,3568 \%$ \\
\hline CPFL PIRATIN & CPFP4 & $17 / 8 / 2005$ & $19,1937 \%$ & $4,8476 \%$ & $24,0413 \%$ & LOCALIZA & RENT3 & $19 / 12 / 2007$ & $3,3719 \%$ & $13,3179 \%$ & $16,6898 \%$ \\
\hline COPEL & CPLE6 & $27 / 4 / 2007$ & $2,9368 \%$ & $7,9439 \%$ & $10,8808 \%$ & RIO GDE ENER & RGEG3 & $4 / 12 / 2007$ & $7,8513 \%$ & $1,7626 \%$ & $9,6139 \%$ \\
\hline SOUZA CRUZ & CRUZ3 & 21/12/2007 & $13,8206 \%$ & $35,5583 \%$ & $49,3789 \%$ & RENNER PART & RNPT4 & $16 / 12 / 2008$ & $7,9275 \%$ & $14,7280 \%$ & $22,6555 \%$ \\
\hline SEG AL BAHIA & CSAB4 & $28 / 2 / 2008$ & $9,8669 \%$ & $11,2658 \%$ & $21,1327 \%$ & INDS ROMI & ROMI3 & $31 / 10 / 2007$ & $7,9801 \%$ & $15,2671 \%$ & $23,2472 \%$ \\
\hline COPASA & CSMG3 & $11 / 9 / 2007$ & $3,4075 \%$ & $11,7165 \%$ & $15,1240 \%$ & ALFA HOLDING & RPAD3 & $17 / 1 / 2008$ & $3,6239 \%$ & $10,2232 \%$ & $13,8471 \%$ \\
\hline SID NACIONAL & CSNA3 & $21 / 12 / 2007$ & $13,2689 \%$ & $14,8164 \%$ & $28,0853 \%$ & PET MANGUINH & RPMG4 & $26 / 1 / 2005$ & $7,1877 \%$ & $14,0847 \%$ & $21,2724 \%$ \\
\hline COSERN & CSRN3 & $18 / 12 / 2007$ & $5,3133 \%$ & $18,2128 \%$ & $23,5261 \%$ & ROSSI RESID & RSID3 & $27 / 4 / 2007$ & $2,1786 \%$ & $6,2125 \%$ & $8,3911 \%$ \\
\hline KARSTEN & CTKA4 & $12 / 12 / 2007$ & $5,5695 \%$ & $8,0278 \%$ & $13,5973 \%$ & RIOSULENSE & RSUL4 & $27 / 4 / 2007$ & $4,8912 \%$ & $15,9950 \%$ & $20,8861 \%$ \\
\hline COTEMINAS & CTNM4 & $30 / 4 / 2007$ & $2,8963 \%$ & $7,4341 \%$ & $10,3304 \%$ & SANEPAR & SAPR4 & $30 / 12 / 2008$ & $8,3504 \%$ & $7,2031 \%$ & $15,5534 \%$ \\
\hline CYRELA REALT & CYRE3 & $29 / 4 / 2008$ & $1,0005 \%$ & $10,7710 \%$ & $11,7715 \%$ & SABESP & SBSP3 & $27 / 2 / 2008$ & $7,6608 \%$ & $6,7185 \%$ & $14,3792 \%$ \\
\hline DOHLER & DOHL4 & $13 / 4 / 2005$ & $4,0915 \%$ & $7,8757 \%$ & $11,9672 \%$ & SAO CARLOS & SCAR3 & $26 / 5 / 2006$ & $2,1090 \%$ & $15,9965 \%$ & $18,1055 \%$ \\
\hline DROGASIL & DROG3 & $13 / 12 / 2007$ & $3,9096 \%$ & $-12,471 \%$ & $-8,5611 \%$ & SADIA S/A & SDIA4 & $6 / 2 / 2008$ & $6,1791 \%$ & $15,4624 \%$ & $21,6415 \%$ \\
\hline MET DUQUE & DUQE4 & $29 / 3 / 2007$ & $7,0813 \%$ & $9,1042 \%$ & $16,1855 \%$ & SERGEN & SGEN4 & $20 / 1 / 2003$ & $2,1428 \%$ & $3,2128 \%$ & $5,3556 \%$ \\
\hline DURATEX & DURA4 & $18 / 2 / 2008$ & $3,5641 \%$ & $8,7596 \%$ & $12,3236 \%$ & SCHULZ & SHUL4 & $28 / 12 / 2007$ & $2,2235 \%$ & $7,1852 \%$ & $9,4087 \%$ \\
\hline DIXIE TOGA & DXTG4 & $30 / 4 / 2007$ & $1,7707 \%$ & $7,5436 \%$ & $9,3143 \%$ & TECEL S JOSE & SJOS4 & $15 / 12 / 2000$ & $6,5591 \%$ & $1,0505 \%$ & $7,6097 \%$ \\
\hline Embratel Part & EBTP4 & $25 / 4 / 2007$ & $2,0172 \%$ & $2,3265 \%$ & $4,3437 \%$ & SARAIVA LIVR & SLED4 & $1 / 3 / 2007$ & $3,1604 \%$ & $13,5232 \%$ & $16,6836 \%$ \\
\hline ELETROBRAS & ELET3 & $2 / 5 / 2007$ & $2,4813 \%$ & $2,6293 \%$ & $5,1105 \%$ & SONDOTECNICA & SOND6 & $28 / 1 / 2008$ & $8,4233 \%$ & $9,2090 \%$ & $17,6323 \%$ \\
\hline EMBRAER & EMBR3 & $17 / 12 / 2007$ & $4,4592 \%$ & $29,5773 \%$ & $34,0366 \%$ & SPRINGER & SPRI3 & $12 / 1 / 2004$ & $15,8016 \%$ & $2,4211 \%$ & $18,2226 \%$ \\
\hline ENERSUL & ENER6 & $23 / 3 / 2005$ & $0,7717 \%$ & $10,8397 \%$ & $11,6114 \%$ & SULTEPA & SULT4 & $4 / 6 / 2007$ & $3,2938 \%$ & $2,9975 \%$ & $6,2913 \%$ \\
\hline ESCELSA & ESCE3 & $30 / 3 / 2005$ & $4,8043 \%$ & $13,2499 \%$ & $18,0541 \%$ & SUZANO PAPEL & SUZB5 & $20 / 12 / 2007$ & $0,0945 \%$ & $17,1740 \%$ & $17,2685 \%$ \\
\hline ETERNIT & ETER3 & $27 / 2 / 2008$ & $21,1813 \%$ & $18,1550 \%$ & $39,3363 \%$ & TRACTEBEL & TBLE3 & $13 / 12 / 2007$ & $8,7010 \%$ & $28,9983 \%$ & $37,6993 \%$ \\
\hline
\end{tabular}


Tabela A-1 (continuação)

\begin{tabular}{|c|c|c|c|c|c|c|c|c|c|c|c|}
\hline Nome de Pregão & Código & Fim (período) & $\mathbf{D}_{1} / \mathbf{P}_{0}$ & G & $\mathbf{R}_{\mathbf{i}}$ & Nome de Pregão & Código & Fim (período) & $\mathbf{D}_{1} / \mathbf{P}_{0}$ & $\mathbf{G}$ & $\mathbf{R}_{\mathbf{i}}$ \\
\hline FIBAM & FBMC4 & $12 / 4 / 2007$ & $13,7978 \%$ & $12,0919 \%$ & $25,8897 \%$ & TECNOSOLO & TCNO4 & $25 / 4 / 2007$ & $1,5775 \%$ & $3,8998 \%$ & $5,4774 \%$ \\
\hline FERBASA & FESA4 & $14 / 12 / 2007$ & $5,6202 \%$ & $10,6852 \%$ & $16,3053 \%$ & TIM PART S/A & TCSL4 & $12 / 4 / 2007$ & $2,3852 \%$ & $10,9412 \%$ & $13,3264 \%$ \\
\hline FOSFERTIL & FFTL4 & $24 / 9 / 2007$ & $11,9700 \%$ & $27,9387 \%$ & $39,9087 \%$ & MILLENNIUM & TIBR5 & $26 / 4 / 2007$ & $5,6302 \%$ & $7,0722 \%$ & $12,7024 \%$ \\
\hline Forjas Taurus & FJTA4 & $27 / 11 / 2007$ & $10,6498 \%$ & $12,2096 \%$ & $22,8594 \%$ & TEKNO & TKNO4 & $21 / 12 / 2007$ & $11,1367 \%$ & $17,5568 \%$ & $28,6934 \%$ \\
\hline FRAS-LE & FRAS4 & $3 / 12 / 2007$ & $6,2118 \%$ & $25,2570 \%$ & $31,4688 \%$ & TELESP & TLPP4 & $28 / 12 / 2007$ & $10,7426 \%$ & $15,5677 \%$ & $26,3102 \%$ \\
\hline GER PARANAP & GEPA3 & $17 / 4 / 2006$ & $9,8884 \%$ & $2,3593 \%$ & $12,2476 \%$ & TELEMAR N L & TMAR5 & $17 / 12 / 2007$ & $8,0345 \%$ & $8,8852 \%$ & $16,9197 \%$ \\
\hline AES TIETE & GETI4 & $20 / 12 / 2007$ & $11,0127 \%$ & $67,7124 \%$ & $78,7250 \%$ & TELEMIG PART & TMCP4 & $27 / 4 / 2007$ & $2,5767 \%$ & $12,4995 \%$ & $15,0763 \%$ \\
\hline GERDAU & GGBR4 & $22 / 2 / 2008$ & $6,0594 \%$ & $23,2384 \%$ & $29,2977 \%$ & TELEMIG CL & TMGC3 & $28 / 11 / 2007$ & $4,2030 \%$ & $18,9933 \%$ & $23,1963 \%$ \\
\hline GLOBEX & GLOB3 & $16 / 4 / 2007$ & $1,0245 \%$ & $6,1007 \%$ & $7,1252 \%$ & TELE NORT CL & TNCP4 & $20 / 2 / 2006$ & $1,2151 \%$ & $10,6000 \%$ & $11,8150 \%$ \\
\hline GERDAU MET & GOAU4 & $22 / 2 / 2008$ & $9,0509 \%$ & $25,1610 \%$ & $34,2118 \%$ & TELEMAR & TNLP4 & $17 / 12 / 2007$ & $4,1927 \%$ & $7,4763 \%$ & $11,6690 \%$ \\
\hline GOL & GOLL4 & $21 / 12 / 2007$ & $2,1342 \%$ & $26,3674 \%$ & $28,5016 \%$ & TRAN PAULIST & TRPL4 & $21 / 1 / 2008$ & $10,3423 \%$ & $5,5563 \%$ & $15,8986 \%$ \\
\hline GUARARAPES & GUAR3 & $18 / 4 / 2007$ & $2,9034 \%$ & $8,3082 \%$ & $11,2116 \%$ & TEX RENAUX & TXRX4 & $14 / 4 / 2005$ & $1,9772 \%$ & $2,1620 \%$ & $4,1392 \%$ \\
\hline IGUACU CAFE & IGUA6 & $28 / 11 / 2007$ & $7,4544 \%$ & $7,2531 \%$ & $14,7075 \%$ & USIN C PINTO & UCOP4 & $16 / 8 / 2007$ & $3,5380 \%$ & $-0,5718 \%$ & $2,9662 \%$ \\
\hline ITAUTEC & ITEC3 & $15 / 2 / 2008$ & $2,4428 \%$ & $10,3130 \%$ & $12,7558 \%$ & ULTRAPAR & UGPA4 & $27 / 2 / 2008$ & $6,6229 \%$ & $15,9540 \%$ & $22,5769 \%$ \\
\hline ITAUSA & ITSA4 & $22 / 2 / 2008$ & $5,5018 \%$ & $19,2473 \%$ & $24,7491 \%$ & UNIPAR & UNIP6 & $10 / 12 / 2007$ & $7,6399 \%$ & $11,3807 \%$ & $19,0206 \%$ \\
\hline JOAO FORTES & JFEN3 & $30 / 4 / 2007$ & $8,8814 \%$ & $6,2064 \%$ & $15,0878 \%$ & USIMINAS & USIM5 & $27 / 12 / 2007$ & $5,9751 \%$ & $19,6872 \%$ & $25,6623 \%$ \\
\hline KEPLER WEBER & KEPL3 & $30 / 3 / 2005$ & $1,6691 \%$ & $13,1418 \%$ & $14,8109 \%$ & VALE R DOCE & VALE5 & $18 / 10 / 2007$ & $5,3098 \%$ & $-48,380 \%$ & $-43,0699 \%$ \\
\hline KLABIN S/A & KLBN4 & $23 / 8 / 2007$ & $4,4302 \%$ & $19,6932 \%$ & $24,1234 \%$ & V C P & VCPA4 & $20 / 12 / 2007$ & $3,7820 \%$ & $15,6839 \%$ & $19,4659 \%$ \\
\hline LOJAS AMERIC & LAME4 & $30 / 11 / 2007$ & $4,7139 \%$ & $38,5294 \%$ & $43,2433 \%$ & VIGOR & VGOR4 & $26 / 4 / 2006$ & $1,8847 \%$ & $1,2541 \%$ & $3,1388 \%$ \\
\hline LECO & LECO4 & $5 / 4 / 2006$ & $2,7675 \%$ & $1,9790 \%$ & $4,7465 \%$ & WEG & WEGE3 & $19 / 2 / 2008$ & $4,1610 \%$ & $23,7707 \%$ & $27,9316 \%$ \\
\hline METAL LEVE & LEVE4 & $4 / 12 / 2007$ & $7,4506 \%$ & $26,5222 \%$ & $33,9728 \%$ & WHIRLPOOL & WHRL4 & $18 / 1 / 2008$ & $5,1747 \%$ & $8,0438 \%$ & $13,2185 \%$ \\
\hline LOJAS RENNER & LREN3 & $27 / 12 / 2007$ & $1,2281 \%$ & $0,8804 \%$ & $2,1085 \%$ & & & & & & \\
\hline
\end{tabular}

